“(C) 2018 IEEE. Personal use of this material is permitted. Permission from IEEE must be obtained for all other uses, in any current or future media, including reprinting/republishing this material for advertising or promotional purposes, creating new collective works, for resale or redistribution to servers or lists, or reuse of any copyrighted component of this work in other works." 


\title{
Performance Analysis of Dense Small Cell Networks with Dynamic TDD
}

\author{
Tian Ding, Student Member, IEEE, Ming Ding, Senior Member, IEEE, Guoqiang Mao, Fellow, IEEE, \\ Zihuai Lin, Senior Member, IEEE, Albert Zomaya, Fellow, IEEE, David López-Pérez, Senior Member, IEEE
}

\begin{abstract}
Small cell networks (SCNs) are envisioned to embrace dynamic time division duplexing (TDD) in order to tailor downlink (DL)/uplink (UL) subframe resources to quick variations and burstiness of DL/UL traffic. The study of dynamic TDD is particularly important because it serves as the predecessor of the full duplex transmission technology, which has been identified as one of the candidate technologies for the 5th-generation (5G) networks. In this paper, we study the performance of the synchronous dynamic TDD from a media access control (MAC) layer viewpoint, which has been widely adopted in the existing 4th-generation (4G) systems. Furthermore, we analyse the coverage probability and the area spectral efficiency (ASE) in the downlink (DL) and uplink (UL) of dense small cell networks (SCNs) considering the synchronous dynamic TDD transmissions, and the performance impact of dynamic TDD transmissions on the ASE in the DL and UL of dense SCNs is discussed. Moreover, the performance impact of interference cancellation (IC) is also explored. Our analytical results shed new light on the performance of dynamic TDD in future synchronous 5G networks.
\end{abstract}

Index Terms-Dynamic time-division duplex (TDD), synchronous networks, media access control (MAC) layer, time resource utilization (TRU), physical (PHY) layer, inter-cell interlink interference.

\section{INTRODUCTION}

In recent years, the increase in mobile data traffic has been shown to project an exponential trajectory, and this trend is expected to continue through the next decade. To meet this formidable traffic demand, telecommunication networks have marched beyond the fourth-generation (4G) realm and begun to explore new advanced technologies [1]. Small cell networks (SCNs) can achieve a high spatial reuse through

Copyright (c) 2015 IEEE. Personal use of this material is permitted. However, permission to use this material for any other purposes must be obtained from the IEEE by sending a request to pubs-permissions@ieee.org.

Tian Ding is with the School of Electrical and Data Engineering, The University of Technology Sydney, Australia (e-mail: Tian.Ding@student.uts.edu.au).

Ming Ding is with Data61, CSIRO, Australia (e-mail: Ming.Ding@data61.csiro.au).

Guoqiang Mao is with the School of Electrical and Data Engineering, The University of Technology Sydney, Australia and Data61, CSIRO, Australia (e-mail: g.mao@ieee.org).

Zihuai Lin is with the School of Electrical and Information Engineering, The University of Sydney, Australia (e-mail: zihuai.lin@sydney.edu.au).

Albert Zomaya is with the School of IT, the University of Sydney, Australia (email: albert.zomaya@sydney.edu.au).

David López-Pérez is with Bell Labs, Nokia, Dublin, Ireland (email: dr.david.lopez@ieee.org). network densification, which in turn can significantly enhance network capacity. Particularly, the orthogonal deployment of SCNs within the existing macrocell network, i.e., small cells and macrocells operating on different frequency spectrum, was prioritized in the design of the 4th generation (4G) Long Term Evolution (LTE) networks by the 3rd Generation Partnership Project (3GPP), and dense SCNs are envisaged to be the workhorse for capacity enhancement in the 5th generation (5G) networks due to its large performance gains and its easy deployment [1].

Besides SCNs, it is also envisaged that $4 \mathrm{G} / 5 \mathrm{G}$ wireless communication networks, e.g., LTE Release 12 14 networks, will embrace time division duplexing (TDD), which does not require a pair of frequency carriers and holds the possibility of tailoring the amount of downlink (DL)/uplink (UL) radio resources to the traffic conditions. In the LTE Release 8 11 networks, seven TDD configurations, each associated with a DL-to-UL subframe ratio in a 10-milisecond transmission frame, are available for semi-static selection at the network side [2]. However, the adopted semi-static selection of TDD configuration in LTE Release 8 11 networks is not able to adapt DL/UL subframe resources to the fast fluctuations in DL/UL traffic loads. These fluctuations are exacerbated in small cells due to the low number of connected UEs per small cell and the burstiness of their DL and UL traffic demands.

In order to allow a more dynamic and independent adaptation of TDD SCNs to the quick variation of DL/UL traffic demands, a new technology, referred to as dynamic TDD, has drawn much attention recently [3]. With dynamic TDD, the configuration of the TDD DL/UL subframe number in each cell or a cluster of cells can be dynamically changed on a perframe basis, i.e., once every 10 milliseconds. Dynamic TDD can thus provide a tailored configuration of DL/UL subframe resources for each cell or a cluster of cells at the expense of allowing inter-cell inter-link interference, e.g., DL transmissions of a cell may interfere with UL ones of a neighboring cell and vice versa. In more detail, dynamic TDD allows each base station (BS) to decide its own UL and DL split. However, this creates two additional sources of interference. First, DL transmissions interfering with UL receivers causing interference on BS to BS links. Second, UL transmissions interfering with DL receivers causing interference on user equipment (UE) to UE links. Characterizing the rate distribution in such networks is important for understanding the trade-off between these new types of interference versus a more flexible resource allocation. The study of dynamic TDD is particularly important because it serves as the predecessor of the full duplex (FD) transmission 
technology, which has been identified as one of the candidate 5G technologies [4].

From the practical views of realistic network deployment, dynamic TDD exploits synchronous frame structures instead of asynchronous ones. More specifically, in an asynchronous network, e.g., Wireless Fidelity (Wi-Fi), the TDD transmission frames are not aligned in time and frequency among cells, i.e., the frame structure is not fixed and each subframe can be DL/UL based on the data requested. Thus the inter-cell interlink interference is statistically uniform in the time domain and only depends on the DL/UL transmission probability. However, in a synchronous network, such as LTE, the TDD transmission frames from different cells are aligned in time and frequency to simplify the protocol design of radio networks [5]. As a result, the inter-cell inter-link interference becomes a function of the LTE TDD configuration structure.

Thus, this paper assumes a scenario of orthogonal deployments of dense SCNs with dynamic TDD, and focuses on studying the system performance. In this paper, we first conduct a theoretical study on the media access control (MAC) layer performance of synchronous dynamic TDD, which has not been investigated from a theoretical viewpoint in the literature. Then we carefully investigate the physical (PHY) layer performance of synchronous dynamic TDD combined with our results on the MAC layer time resource utilization (TRU), with emphasis on the analysis of inter-link interference and UL interference cancellation (IC). Our theoretical study sheds a new light in the interesting question: what is the true value of dynamic TDD?

Compared with existing work, the main contributions of this paper are as follows:

- We derive closed-form expressions of the $D L / U L$ time resource utilization (TRU) for dynamic TDD, which has been widely adopted in the existing LTE systems.

- We derive closed-form expressions for the coverage probability and the area spectral efficiency (ASE) of both the DL and UL, considering dynamic TDD in the synchronous case.

- Based on the above analytical results, the performance of synchronous dynamic TDD is quantified as SCNs evolve into dense ones.

Note that preliminary results of this work has been presented as a conference paper [5]. Compared with [5], the additional contributions of this journal extension are:

- The probability of inter-cell inter-link interference of dynamic TDD is presented in this work.

- The physical (PHY) layer performance analysis of dynamic TDD is thoroughly studied in this work, which was not touched in [5].

- We have combined our results on the MAC layer TRU with the PHY layer SINR results to derive the total area spectral efficiency for synchronous dynamic TDD.

The remainder of this paper is structured as follows. Section II introduces the related work. Section III describes the system model. Section IV presents our main analytical results on the media access control (MAC) layer analysis of the synchronous dynamic TDD. Section $\mathrm{V}$ presents our main analytical results on the UL/DL coverage probability. The simulation and analytical results are discussed in Section VI. Finally, the conclusions are drawn in Section VII.

\section{RELATED WORK}

From the media access control (MAC) layer viewpoint, most of the previous works [6], [7], [8], [9], [10] only investigated the dynamic TDD operating in an asynchronous network, and did not study the performance of the dynamic TDD operating in a synchronous one.

From the physical (PHY) layer viewpoint, the signal-tointerference-plus-noise ratio (SINR) performance of dynamic TDD has been analyzed assuming deterministic positions of BSs and UEs in [11], and considering stochastic positions of BSs and UEs in [6], [7] with the homogeneous poisson point process (HPPP) model. However, it is difficult to directly perform analysis of the inter-cell inter-link interference using the HPPP model. For example, in [6], the authors scaled the BS density in their analysis based on the simulation results of coverage probability, to make them match well. Similar tricks were adopted in [4] to address the complex problem of inter-cell inter-link interference. In [8], the energy efficiency of pico BSs equipped with multi-antenna with dynamic TDD was analyzed based on simulation and analytical results. In [12], the packet throughput of dynamic TDD with random traffic arrivals was analyzed based on simulation and analytical results. In [13], the coverage probability and throughput of dynamic TDD with device-to-device (D2D) were analyzed based on simulation and analytical results. The authors of [8], [12], [13] assumed a small cell scenario, however, the lineof-sight (LoS)/non-line-of-sight (NLoS) pathloss model is not considered, and the performance impact of BS density needs to be discussed in greater details. In [9], the interference performance of dynamic TDD with distributed interference coordination was analyzed based on simulation and analytical results; however, the authors assumed a fully loaded $\mathrm{SCN}$, and the BS idle mode operation was not considered. In [10], the performance impact of self-backhauling with dynamic TDD was analyzed based on simulation and analytical results. Moreover, in [10], the authors assumed a piecewise pathloss model to investigate the performance impact of LoS and NLoS transmission. However, the authors assumed the same pathloss component and LoS probability for all intra-link and inter-link paths, which is over-simpled and a more complicated pathloss model needs to be explored.

\section{SYSTEM MODEL}

\section{A. General Network Scenario}

We consider a cellular network with BSs deployed on a plane according to a homogeneous Poisson point process (HPPP) $\Phi$ with a density of $\lambda \mathrm{BSs} / \mathrm{km}^{2}$. Active UEs are distributed as a HPPP $\Phi_{\mathrm{UE}}$ with density $\lambda_{\mathrm{UE}}$. Here, we only consider active UEs in the network because non-active UEs do not trigger data transmissions. In practice, a BS will mute its transmission, if there is no UE connected to it, which reduces inter-cell interference and energy consumption [14]. Note that such BS idle mode operation is not trivial, which even changes 


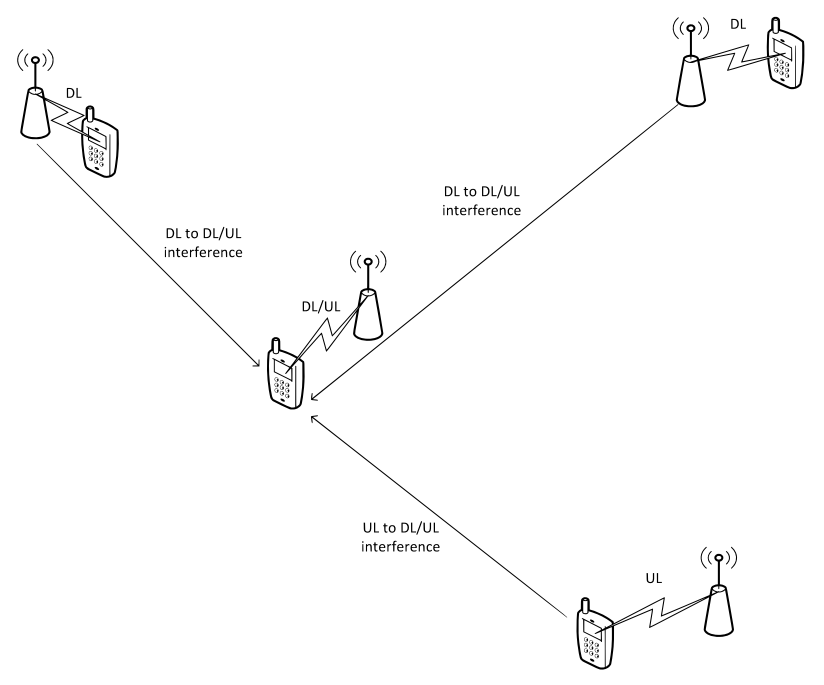

Fig. 1. Dynamic TDD scenarios.

the capacity scaling law [14]. Since UEs are randomly and uniformly distributed in the network, we assume that the active BSs also follow an HPPP distribution $\tilde{\Phi}$, the density of which is denoted by $\tilde{\lambda} \mathrm{BSs} / \mathrm{km}^{2}$. Note that $0 \leq \tilde{\lambda} \leq \lambda$, and a larger $\lambda_{\mathrm{UE}}$ leads to a larger $\tilde{\lambda}$. From [15], [16], $\tilde{\lambda}$ is given by

$$
\tilde{\lambda}=\lambda\left[1-\frac{1}{\left(1+\frac{\lambda_{\mathrm{UE}}}{q \lambda}\right)^{q}}\right],
$$

where $q$ takes an empirical value around 3.5 4 [15], [16].

For each active UE, the probabilities of it requesting DL and UL data are respectively denoted by $p^{\mathrm{D}}$ and $p^{\mathrm{U}}$, with $p^{\mathrm{D}}+p^{\mathrm{U}}=1$. Besides, we assume that each request is large enough to be transmitted for at least one TDD frame, which consists of $T$ subframes. In the sequel, the DL or UL subframe number per frame will be shortened as the DL or UL subframe number, because subframes are meant within one frame.

We focus on the UL of a randomly picked BS from $\Phi$, named the typical BS, and the DL of a randomly picked UE from the UEs served by the typical BS, defined as the typical UE. We assume that at any subframe in any small cell, there is exactly one active transmission, either on the DL with probability $p^{\mathrm{D}}$, or on the UL with probability $p^{\mathrm{U}}$. Then the set of transmitting BSs at the subframe of interest is described by a PPP $\Phi^{\mathrm{D}}$ with density $\lambda^{\mathrm{D}}=\tilde{\lambda} p^{\mathrm{D}}$, and the set of transmitting UEs at the subframe of interest is described by a PPP $\Phi^{\mathrm{U}}$ with density $\lambda^{\mathrm{U}}=\tilde{\lambda} p^{\mathrm{U}}$. The system model is shown in Fig. 1 .

Following [17], we adopt a general and practical path loss model, in which the path loss $\zeta(r)$ associated with distance $r$ is segmented into $N$ pieces written as

$$
\zeta^{D i r}(r)=\left\{\begin{array}{ll}
\zeta_{1}^{D i r}(r), & \text { when } 0 \leq r \leq d_{1} \\
\zeta_{2}^{D i r}(r), & \text { when } d_{1}<r \leq d_{2} \\
\vdots & \vdots \\
\zeta_{N}^{D i r}(r), & \text { when } r>d_{N-1}
\end{array},\right.
$$

where the string variable $\operatorname{Dir}$ denotes the path loss direction and takes the value of 'B2U', 'B2B' and ' $\mathrm{U} 2 \mathrm{U}$ ' for the

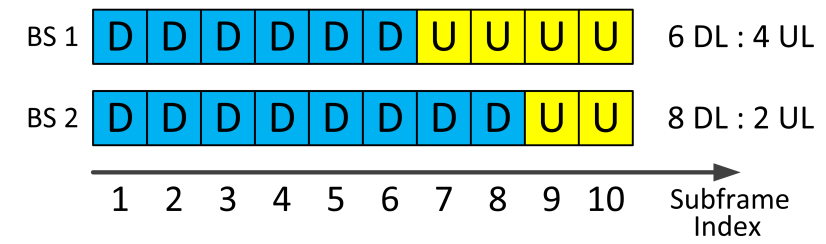

Fig. 2. An example of the LTE TDD configurations. ('D' and 'U' denote a DL subframe and an UL one, respectively.)

BS-to-UE path loss, the BS-to-BS path loss and the UE-toUE path loss, respectively. Besides, each piece $\zeta_{n}^{\text {Dir }}(r), n \in$ $\{1,2, \ldots, N\}$, is modeled as

$$
\zeta_{n}^{D i r}(r)= \begin{cases}\zeta_{n}^{D i r, \mathrm{~L}}(r)=\frac{A_{n}^{D i r, \mathrm{~L}}}{r^{\alpha_{n}^{i}, \mathrm{~L}},}, & \mathrm{LoS}: \operatorname{Pr}_{n}^{D i r, \mathrm{~L}}(r) \\ \zeta_{n}^{D i r, \mathrm{NL}}(r)=\frac{A_{n}^{D i r, \mathrm{NL}}}{r^{\alpha_{n}^{D i r, \mathrm{NL}}},} & \mathrm{NLOS}: 1-\operatorname{Pr}_{n}^{D i r, \mathrm{~L}}(r)^{\prime}\end{cases}
$$

where $\zeta_{n}^{D i r, \mathrm{~L}}(r)$ and $\zeta_{n}^{D i r, \mathrm{NL}}(r)$ are the $n$-th piece path loss functions for the LoS transmission and the NLoS transmission, respectively, $A_{n}^{D i r, \mathrm{~L}}$ and $A_{n}^{D i r, \mathrm{NL}}$ are the path losses at a reference distance $r=1$ for the LoS and the NLoS cases, respectively, and $\alpha_{n}^{D i r, \mathrm{~L}}$ and $\alpha_{n}^{D i r, \mathrm{NL}}$ are the path loss exponents for the LoS and the NLoS cases, respectively. In practice, $A_{n}^{D i r, \mathrm{~L}}, A_{n}^{D i r, \mathrm{NL}}, \alpha_{n}^{D i r, \mathrm{~L}}$ and $\alpha_{n}^{D i r, \mathrm{NL}}$ are constants obtainable from field tests [2].

Moreover, $\operatorname{Pr}_{n}^{D i r, \mathrm{~L}}(r)$ is the $n$-th piece LoS probability function for the event that there is a LoS path between a transmitter and a receiver separated by a distance $r$.

In this paper, we assume a practical user association strategy (UAS), in which each UE is connected to the BS with the strongest received signal strength (i.e., with the largest $\zeta(r))$ [17]. Moreover, we assume that each BS/UE is equipped with an isotropic antenna, and that the multi-path fading between a transmitter and a receiver is modeled as independent and identically distributed (i.i.d.) Rayleigh fading [17]. Note we have found in previous work that a general multi-path fading model based on Rician fading has a minor impact on the performance of SCNs, i.e., change the results quantitatively but not qualitatively, and thus its incorporation into theoretical analyses is less urgent [18].

\section{B. Synchronous Networks}

Regarding TDD frames, we focus on the analysis of the synchronous networks. Fig. 2 shows an example of such TDD configuration structure in LTE with $T=10$ [2]. In Fig. 2, TDD frames of different cells are aligned in the time domain. One TDD frame is composed of $T=10$ subframes, and the time length of each subframe is 1 millisecond [2]. As an example, we assume that BS 1 and BS 2 use the TDD configurations with 6 and 8 DL subframes, respectively. Note that the current LTE behaviour in dynamic TDD is first DL and then UL. For this synchronous network with TDD frame alignment, we have the following two remarks.

Remark 1: The first few subframes are more likely to carry DL transmissions than the last few ones. The opposite conclusion applies for the UL. This leads to a subframe dependent MAC layer performance of dynamic TDD. 
Remark 2: The subframes in the middle of the frame are more likely to be subject to inter-cell inter-link interference than those at the beginning and at the end. This implies a subframe dependent PHY layer performance of dynamic TDD.

In this paper, we explore these two Remarks, and study the system performance of synchronous dynamic TDD, with the consideration of the DL-before-UL TDD configuration structure adopted in LTE (see Fig. 2).

\section{Performance Metrics}

1) MAC-layer Performance Metrics: For the l-th subframe $(l \in\{1,2, \ldots, T\})$, we define the subframe dependent DL TRU and UL TRU as the probability of the BS transmitting DL signals and that of UEs transmitting UL signals, respectively, which are denoted by $q_{l}^{\mathrm{D}}$ and $q_{l}^{\mathrm{U}}$. In other words, $q_{l}^{\mathrm{D}}$ and $q_{l}^{\mathrm{U}}$ characterize how much time resource is actually used for DL and UL, respectively. Note that we may or may not have $q_{l}^{\mathrm{D}}+q_{l}^{\mathrm{U}}=1$, depending on whether we have a dynamic enough TDD and the traffic conditions.

Moreover, we define the average DL TRU $\kappa^{\mathrm{D}}$ and the average UL TRU $\kappa^{\mathrm{U}}$ as the mean value of $q_{l}^{\mathrm{D}}$ and $q_{l}^{\mathrm{U}}$ across all of the $T$ subframes:

$$
\left\{\begin{array}{l}
\kappa^{\mathrm{D}}=\frac{1}{T} \sum_{l=1}^{T} q_{l}^{\mathrm{D}} \\
\kappa^{\mathrm{U}}=\frac{1}{T} \sum_{l=1}^{T} q_{l}^{\mathrm{U}}
\end{array} .\right.
$$

Finally, we define the average total TRU $\kappa$ as the sum of $\kappa^{\mathrm{D}}$ and $\kappa^{\mathrm{U}}$, which is written as

$$
\kappa=\kappa^{\mathrm{D}}+\kappa^{\mathrm{U}} .
$$

In the following sections, we will investigate the performance of $q_{l}^{\text {Link }}, \kappa^{\text {Link }}$ and $\kappa$ considering Remark 1, where the string variable Link denotes the link direction and takes the value of ' $D$ ' and ' $U$ ' for the DL and the UL, respectively.

2) PHY-layer Performance Metrics: Based on the system model presented in Subsection III-A, we can define the coverage probability that the typical UE's DL/UL SINR is above a designated threshold $\gamma$ as

$$
p^{\mathrm{cov}, \text { Link }}(\lambda, \gamma)=\operatorname{Pr}\left[\operatorname{SINR}^{\text {Link }}>\gamma\right] .
$$

Moreover, the DL/UL SINR is calculated by

$$
\mathrm{SINR}^{\text {Link }}=\frac{P^{\text {Link }} \zeta_{b_{o}}^{\mathrm{B} 2 \mathrm{U}}(r) h}{I_{\mathrm{agg}}^{\text {Link, }}+I_{\mathrm{agg}}^{\text {Link,U }}+P_{\mathrm{N}}^{\text {Link }}},
$$

where $P^{\text {Link }}$ is the transmission power, $r$ is the distance from the typical UE to the typical BS denoted by $b_{o}, h$ is the Rayleigh channel gain modeled as an exponentially distributed random variable (RV) with a mean of one as mentioned above, $P_{\mathrm{N}}^{L i n k}$ is the additive white Gaussian noise (AWGN) power, and $I_{\mathrm{agg}}^{L i n k, \mathrm{D}}\left(I_{\mathrm{agg}}^{L i n k, \mathrm{U}}\right)$ is the DL (UL) cumulative interference, respectively. It is important to note that:

- For the DL, $P^{\text {Link }}$ takes the value of the BS power, i.e., $P^{\mathrm{D}}$, which is usually a cell-specific constant to maintain a stable DL coverage [2]. Besides, $P_{\mathrm{N}}^{\text {Link }}$ should be the AWGN at the UE side, i.e., $P_{\mathrm{N}}^{\mathrm{D}}$.

- For the UL, $P^{\text {Link }}$ takes the value of the UE power, i.e., $P^{\mathrm{U}}$, which is assumed as a constant in this paper [2].
Table I

NOTATION OF VARIABLES

\begin{tabular}{|c|c|}
\hline Notation & Items \\
\hline \hline$q_{l}^{\mathrm{D}}, q_{l}^{\mathrm{U}}$ & Subframe dependent DL TRU and UL TRU \\
\hline$\kappa, \kappa^{\mathrm{D}}, \kappa^{\mathrm{U}}$ & Average total TRU, DL TRU and UL TRU \\
\hline$P^{\mathrm{D}}, P^{\mathrm{U}}$ & DL and UL transmission power \\
\hline$I_{\mathrm{agg}}^{L i n k, \mathrm{D}}, I_{\mathrm{agg}}^{L i n k, \mathrm{U}}$ & DL and UL cumulative interference \\
\hline$P_{\mathrm{N}}^{\mathrm{D}}, P_{\mathrm{N}}^{\mathrm{U}} \cdot$ & DL and UL AWGN power \\
\hline
\end{tabular}

Besides, $P_{\mathrm{N}}^{\text {Link }}$ should be the AWGN at the BS side, i.e., $P_{\mathrm{N}}^{\mathrm{U}}$.

- Due to the existence of inter-cell inter-link interference, $I_{\text {agg }}^{\mathrm{D}}$ and $I_{\text {agg }}^{\mathrm{U}}$ are probabilistic interference emitted from interfering BSs and interfering UEs, respectively.

For clarity, the notation of variables is summarized in Table I.

3) Combined Performance Metrics: According to [17], the SINR-dependent DL/UL area spectral efficiency (ASE) in $\mathrm{bps} / \mathrm{Hz} / \mathrm{km}^{2}$ can be defined by

$$
A S E^{L i n k}\left(\lambda, \gamma_{0}\right)=\kappa^{L i n k} \lambda \int_{\gamma_{0}}^{+\infty} \log _{2}(1+\gamma) f_{\Gamma}^{L i n k}(\lambda, \gamma) d \gamma,(8)
$$

where $\kappa^{\text {Link }}$ is the DL/UL time resource utilization characterizing how much time resource is actually used for the DL/UL, $\gamma_{0}$ is the minimum working SINR for the considered SCN, and $f_{\Gamma}^{L i n k}(\lambda, \gamma)$ is the probability density function (PDF) of SINR $^{\text {Link }}$ at a particular BS density $\lambda$.

Based on the definition of $p^{\text {cov, Link }}(\lambda, \gamma)$ in (6), which is the complementary cumulative distribution function (CCDF) of the DL/UL SINR, $f_{\Gamma}^{\operatorname{Link}}(\lambda, \gamma)$ can be computed by

$$
f_{\Gamma}^{\text {Link }}(\lambda, \gamma)=\frac{\partial\left(1-p^{\text {cov }, \text { Link }}(\lambda, \gamma)\right)}{\partial \gamma} .
$$

\section{Main Results of the MAC Layer Analysis}

The main goal of this section is to derive theoretical results on the DL/UL TRU defined in Subsection III-C. Note that computing $q_{l}^{\mathrm{D}}$ and $q_{l}^{\mathrm{U}}$ is a non-trivial task for synchronous dynamic TDD, because it involves the following distributions:

- the distribution of the UE number in an active BS, which will be shown to follow a truncated Negative Binomial distribution in Subsection IV-A;

- the distribution of the DL/UL data request numbers in an active BS, which will be shown to follow a Binomial distribution in Subsection IV-B;

- the dynamic TDD subframe splitting strategy and the corresponding distribution of the DL/UL subframe number, which will be shown to follow an aggregated Binomial distribution in Subsection IV-C; and finally

- the prior information about the TDD frame structure, such as the DL-before-UL structure adopted in LTE [2] (see Fig. 2), which will lead to subframe dependent results of $q_{l}^{\mathrm{D}}$ and $q_{l}^{\mathrm{U}}$ to be presented in Subsection IV-D. 
A. The Distribution of the UE Number in an active BS: A Truncated Negative Binomial Distribution

According to [15], the per-BS coverage area size $X$ can be characterized by a Gamma distribution [15], [19], [20], and the probability density function (PDF) of $X$ can be expressed as

$$
f_{X}(x)=(q \lambda)^{q} x^{q-1} \frac{\exp (-q \lambda x)}{\Gamma(q)},
$$

where $\Gamma(\cdot)$ is the Gamma function [21]. Note that the Gamma approximation was shown to be very accurate in [15], assuming a nearest-distance UAS, where an empirical value of 3.5 was suggested for $q$. In this work, a more realistic received signal strength based UAS is adopted, and thus the corresponding result in [15] cannot be directly applied. Instead, we need to derive a new approximation for the adopted UAS considering probabilistic LoS and NLoS transmissions. Intuitively speaking, from a typical UE's point of view, the equivalent BS density of the considered UAS based on probabilistic LoS and NLoS transmissions should be larger than that of the nearestdistance UAS based on single-slope path loss transmissions. In other words, the existence of LoS BSs provides more candidate BSs for a typical UE to connect with, and thus the equivalent BS density increases for each UE. We have proved that based on the considered path loss model and the adopted UAS, the per-BS coverage area size can be approximated tightly with the gamma approximation in (10) [14]. Moreover, the value of $q$ in (10) is obtained according to the derived lower bound and the upper bound presented in Theorems 3 and 4 of [14], respectively.

Then, the UE number per BS can be denoted by a random variable (RV) $K$, and the probability mass function (PMF) of $K$ can be derived as

$$
\begin{aligned}
f_{K}(k) & =\operatorname{Pr}[K=k] \\
& \stackrel{(a)}{=} \int_{0}^{+\infty} \frac{(\rho x)^{k}}{k !} \exp (-\rho x) f_{X}(x) d x \\
& \stackrel{(b)}{=} \frac{\Gamma(k+q)}{\Gamma(k+1) \Gamma(q)}\left(\frac{\rho}{\rho+q \lambda}\right)^{k}\left(\frac{q \lambda}{\rho+q \lambda}\right)^{q},
\end{aligned}
$$

where $(a)$ is due to the HPPP distribution of UEs and $(b)$ is obtained from (10). It can be seem from (11) that $K$ follows a Negative Binomial distribution [21], i.e., $K \sim \mathrm{NB}\left(q, \frac{\rho}{\rho+q \lambda}\right)$.

As discussed in Subsection III-A, we assume that a BS with $K=0$ is not active, which will be ignored in our analysis due to its muted transmission. Hence, we focus on the active BSs and further study the distribution of the UE number per active $B S$, which is denoted by a positive RV $\tilde{K}$. Considering (11) and the fact that the only difference between $K$ and $\tilde{K}$ lies in $\tilde{K} \neq 0$, we can conclude that $\tilde{K}$ follows a truncated Negative Binomial distribution, i.e., $\tilde{K} \sim \operatorname{truncNB}\left(q, \frac{\rho}{\rho+q \lambda}\right)$. More specifically, the PMF of $\tilde{K}$ is denoted by $f_{\tilde{K}}(\tilde{k}), \tilde{k} \in$ $\{1,2, \ldots,+\infty\}$, and it is given by

$$
f_{\tilde{K}}(\tilde{k})=\operatorname{Pr}[\tilde{K}=\tilde{k}]=\frac{f_{K}(\tilde{k})}{1-f_{K}(0)},
$$

where the denominator $\left(1-f_{K}(0)\right)$ represents the probability of a BS being active, i.e., $\tilde{\lambda} / \lambda$.

\section{B. The Distribution of the DL/UL Data Request Number in an Active BS: A Binomial Distribution}

After obtaining $f_{\tilde{K}}(\tilde{k})$, we need to further study the distribution of the DL/UL data request number in an active BS, so that a tailored TDD configuration can be determined in a dynamic TDD network.

For clarity, the DL and UL data request numbers in an active BS are denoted by RVs $M^{\mathrm{D}}$ and $M^{\mathrm{U}}$, respectively. Since we assume that each UE generates one request of either DL data or UL data (see Subsection III-A), it is easy to show that

$$
M^{\mathrm{D}}+M^{\mathrm{U}}=\tilde{K} .
$$

As discussed in Subsection III-A, for each UE in an active BS, the probability of it requesting DL data and UL data is $p^{\mathrm{D}}$ and $p^{\mathrm{U}}$, respectively. Hence, for a given UE number $\tilde{k}, M^{\mathrm{D}}$ and $M^{\mathrm{U}}$ follow Binomial distributions [21], i.e., $M^{\mathrm{D}} \sim \mathrm{Bi}\left(\tilde{k}, p^{\mathrm{D}}\right)$ and $M^{\mathrm{U}} \sim \mathrm{Bi}\left(\tilde{k}, p^{\mathrm{U}}\right)$. More specifically, the PMFs of $M^{\mathrm{D}}$ and $M^{\mathrm{U}}$ can be respectively written as

$$
f_{M^{\mathrm{D}}}\left(m^{\mathrm{D}}\right)=\left(\begin{array}{c}
\tilde{k} \\
m^{\mathrm{D}}
\end{array}\right)\left(p^{\mathrm{D}}\right)^{m^{\mathrm{D}}}\left(1-p^{\mathrm{D}}\right)^{\tilde{k}-m^{\mathrm{D}}},
$$

and

$$
f_{M^{\mathrm{U}}}\left(m^{\mathrm{U}}\right)=\left(\begin{array}{c}
\tilde{k} \\
m^{\mathrm{U}}
\end{array}\right)\left(p^{\mathrm{U}}\right)^{m^{\mathrm{U}}}\left(1-p^{\mathrm{U}}\right)^{\tilde{k}-m^{\mathrm{U}}} .
$$

\section{The Distribution of the DL/UL Subframe Number with Dynamic TDD: An Aggregated Binomial Distribution}

After knowing the distribution of the DL data request number $M^{\mathrm{D}}$ in an active $\mathrm{BS}$, we are now ready to consider dynamic TDD, and derive the distribution of the DL subframe number in an active BS. For a given UE number $\tilde{k}$, the DL subframe number in an active BS is denoted by $N^{\mathrm{D}}$. Here, we adopt a dynamic TDD algorithm to choose the DL subframe number, which matches the DL subframe ratio with the DL data request ratio [22]. In more detail, for certain values of $m^{\mathrm{D}}$ and $\tilde{k}$, the DL subframe number $n\left(m^{\mathrm{D}}, \tilde{k}\right)$ is determined by

$$
n\left(m^{\mathrm{D}}, \tilde{k}\right)=\operatorname{round}\left(\frac{m^{\mathrm{D}}}{\tilde{k}} T\right),
$$

where round $(x)$ is an operator that rounds a real value $x$ to its nearest integer. In (16), $\frac{m^{\mathrm{D}}}{\tilde{k}}$ can be deemed as the DL data request ratio, because $(i) m^{\mathrm{D}}$ denotes the DL data request number with its distribution characterized in (14); and (ii) $\tilde{k}$ represents the UE number, and thus the total number of the DL and UL data requests. As a result, $\frac{m^{\mathrm{D}}}{\tilde{k}} T$ yields the desirable DL subframe number that matches the DL subframe ratio with the DL data request ratio. Due to the integer nature of the DL subframe number, we use the round operator to generate a valid DL subframe number that is nearest to $\frac{m^{\mathrm{D}}}{\tilde{k}} T$ in (16). 
Based on (16), the PMF of $N^{\mathrm{D}}$ is denoted by $f_{N^{\mathrm{D}}}\left(n^{\mathrm{D}}\right), n^{\mathrm{D}} \in\{0,1, \ldots, T\}$, and it can be derived as

$$
\begin{aligned}
f_{N^{\mathrm{D}}}\left(n^{\mathrm{D}}\right) & =\operatorname{Pr}\left[N^{\mathrm{D}}=n^{\mathrm{D}}\right] \\
& \stackrel{(a)}{=} \sum_{m^{\mathrm{D}}=0}^{\tilde{k}} I\left\{\text { round }\left(\frac{m^{\mathrm{D}}}{\tilde{k}} T\right)=n^{\mathrm{D}}\right\} f_{M^{\mathrm{D}}}\left(m^{\mathrm{D}}\right),
\end{aligned}
$$

where (16) is plugged into (a), and $I\{X\}$ is an indicator function that outputs one when $X$ is true and zero otherwise. Besides, $f_{M^{\mathrm{D}}}\left(m^{\mathrm{D}}\right)$ is computed by (14). Due to the existence of the indicator function in (17), $f_{N^{\mathrm{D}}}\left(n^{\mathrm{D}}\right)$ can be viewed as an aggregated PMF of Binomial PMFs, since $N^{\mathrm{D}}$ is computed from $M^{\mathrm{D}}$ according to a many-to-one mapping in (16).

Because the total subframe number in a frame is $T$, and each subframe should be either a DL one or an UL one, it is apparent that $N^{\mathrm{D}}+N^{\mathrm{U}}=T$, and thus we have

$$
f_{N^{\mathrm{U}}}\left(n^{\mathrm{U}}\right)=f_{N^{\mathrm{D}}}\left(T-n^{\mathrm{U}}\right) \text {. }
$$

\section{The Subframe Dependent DL/UL TRU}

In this Subsection, we present our main results on the subframe dependent DL/UL TRUs $q_{l}^{\mathrm{D}}$ and $q_{l}^{\mathrm{U}}$ for dynamic TDD in Theorem 1.

Theorem 1. For dynamic TDD, $q_{l}^{\mathrm{D}}$ and $q_{l}^{\mathrm{U}}$ are given by

$$
\left\{\begin{array}{l}
q_{l}^{\mathrm{D}}=\sum_{\tilde{k}=1}^{+\infty}\left(1-\sum_{i=0}^{l-1} f_{N^{\mathrm{D}}}(i)\right) f_{\tilde{K}}(\tilde{k}) \\
q_{l}^{\mathrm{U}}=\sum_{\tilde{k}=1}^{+\infty} \sum_{i=0}^{l-1} f_{N^{\mathrm{D}}}(i) f_{\tilde{K}}(\tilde{k})
\end{array},\right.
$$

where $f_{N \mathrm{D}}(i)$ and $f_{\tilde{K}}(\tilde{k})$ are given by (17) and (12), respectively.

Proof: See Appendix A.

\section{E. The Probabilities of Inter-Cell Inter-Link Interference}

With the knowledge on the probability of each subframe being a DL one or an UL one, we can conduct an interesting study on the probabilities of inter-cell inter-link interference for dynamic TDD in the synchronous case. For clarify, such probabilities are formally defined as follows:

- The probability of the DL-to-UL interference is denoted by $\operatorname{Pr}^{\mathrm{D} 2 \mathrm{U}}$ and defined as $\operatorname{Pr}\left[Z={ }^{\prime} \mathrm{D} ' \mid S={ }^{\prime} \mathrm{U}^{\prime}\right]$, where $Z$ and $S$ denote the link directions for the interference and the signal, respectively. Note that the probability of the UL-to-UL interference is denoted by $\operatorname{Pr}^{\mathrm{U} 2 \mathrm{U}}$ and defined as $\operatorname{Pr}\left[Z={ }^{\prime} \mathrm{U}\right.$ ' $\mid S={ }^{\prime} \mathrm{U}$ ']. From the definition of $\operatorname{Pr}^{\mathrm{D} 2 \mathrm{U}}$ and $\operatorname{Pr}^{\mathrm{U} 2 \mathrm{U}}$, we have $\operatorname{Pr}^{\mathrm{D} 2 \mathrm{U}}+\operatorname{Pr}^{\mathrm{U} 2 \mathrm{U}}=1$.

- Similarly, the probability of the UL-to-DL interference is defined by $\operatorname{Pr}^{\mathrm{U} 2 \mathrm{D}} \triangleq \operatorname{Pr}\left[Z={ }^{\prime} \mathrm{U}^{\prime} \mid S={ }^{\prime} \mathrm{D}^{\prime}\right]$. Besides, the probability of the DL-to-DL interference is defined by $\operatorname{Pr}^{\mathrm{D} 2 \mathrm{D}} \triangleq \operatorname{Pr}\left[Z={ }^{\prime} \mathrm{D}^{\prime} \mid S=\right.$ 'D'], with $\operatorname{Pr}^{\mathrm{U} 2 \mathrm{D}}+\operatorname{Pr}^{\mathrm{D} 2 \mathrm{D}}=1$.

Our main results on $\operatorname{Pr}^{\mathrm{D} 2 \mathrm{U}}$ and $\operatorname{Pr}^{\mathrm{U} 2 \mathrm{D}}$ are summarized in Theorem 2.
Theorem 2. $\operatorname{Pr}^{\mathrm{D} 2 \mathrm{U}}$ and $\operatorname{Pr}^{\mathrm{U} 2 \mathrm{D}}$ can be derived in closed-form expressions as

$$
\left\{\begin{array}{l}
\operatorname{Pr}^{\mathrm{D} 2 \mathrm{U}}=\frac{\sum_{l=1}^{T} q_{l}^{\mathrm{D}} q_{l}^{\mathrm{U}}}{\sum_{j=1}^{T} q_{j}^{\mathrm{U}}} \\
\operatorname{Pr}^{\mathrm{U} 2 \mathrm{D}}=\frac{\sum_{l=1}^{T} q_{l}^{\mathrm{U}} q_{l}^{\mathrm{D}}}{\sum_{j=1}^{T} q_{j}^{\mathrm{D}}}
\end{array}\right.
$$

where $q_{l}^{\mathrm{D}}$ and $q_{l}^{\mathrm{U}}$ are obtained from (19).

Proof: See Appendix B.

As discussed at the beginning of Section IV, for static TDD, we have $\operatorname{Pr}^{\mathrm{D} 2 \mathrm{U}}=\operatorname{Pr}^{\mathrm{U} 2 \mathrm{D}}=0$ since all the TDD subframes are of the same sequence and well-aligned. For dynamic TDD in the asynchronous case, we have $\operatorname{Pr}^{\mathrm{D} 2 \mathrm{U}}=p^{\mathrm{D}}$ and $\operatorname{Pr}^{\mathrm{U} 2 \mathrm{D}}=p^{\mathrm{U}}$ [7], due to the random collision of the dynamic TDD subframes. On the other hand, as shown in Theorem 2, $\operatorname{Pr}^{\mathrm{D} 2 \mathrm{U}}$ and $\operatorname{Pr}^{\mathrm{U} 2 \mathrm{D}}$ for dynamic TDD in the synchronous case are much more complex, which are expected to have a major impact on the evaluation of $I_{\text {agg }}^{\mathrm{D}}$ and $I_{\text {agg }}^{\mathrm{U}}$ in (7).

\section{F. The Average DL/UL/Total TRU}

From Theorem 1, and Equations (4) and (5), we can obtain the results on the average DL/UL/total TRU for dynamic TDD, which are summarized in Lemma 3.

Lemma 3. For dynamic $T D D,\left\{\kappa^{\mathrm{D}}, \kappa^{\mathrm{U}}, \kappa\right\}$ is given by

$$
\left\{\begin{array}{l}
\kappa^{\mathrm{D}}=\frac{1}{T} \sum_{l=1}^{T} \sum_{\tilde{k}=1}^{+\infty}\left(1-\sum_{i=0}^{l-1} f_{N^{\mathrm{D}}}(i)\right) f_{\tilde{K}}(\tilde{k}) \\
\kappa^{\mathrm{U}}=\frac{1}{T} \sum_{l=1}^{T} \sum_{\tilde{k}=1}^{+\infty} \sum_{i=0}^{l-1} f_{N^{\mathrm{D}}}(i) f_{\tilde{K}}(\tilde{k}) \\
\kappa=1
\end{array},\right.
$$

where $f_{N^{\mathrm{D}}}(i)$ and $f_{\tilde{K}}(\tilde{k})$ are given by (17) and (12), respectively.

Proof: The proof is straightforward by plugging (19) into (4) and (5).

Lemma 3 not only quantifies the average MAC layer performance of dynamic TDD, but also shows from a theoretical viewpoint that dynamic TDD can always achieve a full resource utilization, thanks to the smart adaption of DL/UL subframes to DL/UL data requests.

Next, we present our main results on $\kappa^{\mathrm{D}}, \kappa^{\mathrm{U}}$ and $\kappa$ for static TDD in Theorem 4.

Theorem 4. For static TDD, $\left\{\kappa^{\mathrm{D}}, \kappa^{\mathrm{U}}, \kappa\right\}$ can be derived as

$$
\left\{\begin{array}{l}
\kappa^{\mathrm{D}}=\left(1-\sum_{\tilde{k}=1}^{+\infty}\left(1-p^{\mathrm{D}}\right)^{\tilde{k}} f_{\tilde{K}}(\tilde{k})\right) \frac{N_{0}^{\mathrm{D}}}{T} \\
\kappa^{\mathrm{U}}=\left(1-\sum_{\tilde{k}=1}^{+\infty}\left(1-p^{\mathrm{U}}\right)^{\tilde{k}} f_{\tilde{K}}(\tilde{k})\right) \frac{N_{0}^{\mathrm{U}}}{T} \\
\kappa=\frac{1}{T} \sum_{\tilde{k}=1}^{+\infty}\left[\left(1-\left(p^{\mathrm{U}}\right)^{\tilde{k}}\right) N_{0}^{\mathrm{D}}+\left(1-\left(p^{\mathrm{D}}\right)^{\tilde{k}}\right) N_{0}^{\mathrm{U}}\right] f_{\tilde{K}}(\tilde{k})
\end{array}\right.
$$

where $f_{\tilde{K}}(\tilde{k})$ is obtained from (12), and $N_{0}^{\mathrm{D}}$ and $N_{0}^{\mathrm{U}}$ are the designated subframe numbers for the $D L$ and the $U L$ in static TDD, respectively, which satisfy $N_{0}^{\mathrm{D}}+N_{0}^{\mathrm{U}}=T$.

Proof: See Appendix C. 
From Lemma 3 and Theorem 4, we can further quantify the additional total TRU achieved by dynamic TDD as

$$
\kappa^{\mathrm{ADD}}=\frac{1}{T} \sum_{\tilde{k}=1}^{+\infty}\left[\left(p^{\mathrm{U}}\right)^{\tilde{k}} N_{0}^{\mathrm{D}}+\left(p^{\mathrm{D}}\right)^{\tilde{k}} N_{0}^{\mathrm{U}}\right] f_{\tilde{K}}(\tilde{k})
$$

where $\kappa^{\mathrm{ADD}}$ measures the difference of $\kappa$ in $(21)$ and that in (22).

In addition, we present the performance limit of $\kappa^{\mathrm{ADD}}$ in Lemma 5.

Lemma 5. When $\lambda \rightarrow+\infty$, the limit of $\kappa^{\mathrm{ADD}}$ is given by

$$
\lim _{\lambda \rightarrow+\infty} \kappa^{\mathrm{ADD}}=\frac{p^{\mathrm{D}} N_{0}^{\mathrm{U}}}{T}+\frac{p^{\mathrm{U}} N_{0}^{\mathrm{D}}}{T} .
$$

Proof:

From (12), we have $\lim _{\lambda \rightarrow+\infty} \operatorname{Pr}[\tilde{K}=1]=1$. Hence, using Lemma 3, we can draw the following conclusion for dynamic TDD:

$$
\left\{\begin{array}{l}
\lim _{\lambda \rightarrow+\infty} \kappa^{\mathrm{D}}=1-f_{N^{\mathrm{D}}}(0)=1-p^{\mathrm{U}}=p^{\mathrm{D}} \\
\lim _{\lambda \rightarrow+\infty} \kappa^{\mathrm{U}}=1-f_{N^{\mathrm{U}}}(0)=1-p^{\mathrm{D}}=p^{\mathrm{U}}
\end{array}\right.
$$

Based on $\lim _{\lambda \rightarrow+\infty} \operatorname{Pr}[\tilde{K}=1]=1$ and Theorem 4, we can obtain the following conclusion for static TDD:

$$
\left\{\begin{array}{l}
\lim _{\lambda \rightarrow+\infty} \kappa^{\mathrm{D}}=\left(1-\left(1-p^{\mathrm{D}}\right)\right) \frac{N_{0}^{\mathrm{D}}}{T}=\frac{p^{\mathrm{D}} N_{0}^{\mathrm{D}}}{T} \\
\lim _{\lambda \rightarrow+\infty} \kappa^{\mathrm{U}}=\left(1-\left(1-p^{\mathrm{U}}\right)\right) \frac{N_{0}^{\mathrm{U}}}{T}=\frac{p^{\mathrm{U}} N_{0}^{\mathrm{U}}}{T}
\end{array} .\right.
$$

Our proof is completed by comparing (25) with (26).

Note that in (24) of Lemma 5, the first and the second terms are contributed from the DL and the UL, respectively.

\section{Main Results of The PHY LAyER ANALYSis}

The main goal of this section is to derive theoretical results on the DL/UL coverage probability defined in Subsection III-C. Note that computing $p^{\text {cov, } \mathrm{D}}(\lambda, \gamma)$ and $p^{\text {cov, } \mathrm{U}}(\lambda, \gamma)$ is a non-trivial task for dynamic TDD, because it involves the following characteristics:

- Two new kinds of inter-cell inter-link interference, i.e., the DL-to-UL and UL-to-DL interference, are introduced in the analysis of the coverage probability. For accurate performance analysis, the dependent relationship between interfering $\mathrm{BS}$ and typical BS, as well as that between interfering UE and typical UE, should be considered carefully. The details will be shown in Subsection V-A.

- The UL performance is the bottleneck of dynamic TDD and we need to manage the detrimental DL-to-UL interference. The UL coverage probability with full IC will be derived in Subsection V-B. Note that having results cancelling a subset of interferers would be ideal, and other interference mitigation methods exist, e.g., clustering, UL power boosting, etc., which are left as possible future work.

- The theoretical analysis of the coverage probability will be applied to a 3GPP special case, and the results will be shown in Subsection V-C.

\section{A. Coverage Probability without Interference Cancellation}

Based on the system model presented in Section III, we can calculate coverage probability $p^{\operatorname{cov}, \operatorname{Link}}(\lambda, \gamma)$ and present it in the following theorem.

Theorem 6. $p^{\mathrm{cov}, \text { Link }}(\lambda, \gamma)$ can be derived as

$$
p^{\operatorname{cov}, \text { Link }}(\lambda, \gamma)=\sum_{n=1}^{N}\left(T_{n}^{\mathrm{L}, \text { Link }}+T_{n}^{\mathrm{NL}, \text { Link }}\right),
$$

where

$$
\begin{aligned}
& T_{n}^{\mathrm{L}, \text { Link }}=\int_{d_{n-1}}^{d_{n}} \operatorname{Pr}\left[\mathrm{SINR}^{\text {Link }}>\gamma \mid r, \mathrm{LoS}\right] f_{R, n}^{\mathrm{L}}(r) d r, \\
& T_{n}^{\mathrm{NL}, \text { Link }}=\int_{d_{n-1}}^{d_{n}} \operatorname{Pr}\left[\mathrm{SINR}^{\text {Link }}>\gamma \mid r, \mathrm{NLoS}\right] f_{R, n}^{\mathrm{NL}}(r) d r,
\end{aligned}
$$

and $d_{0}$ and $d_{N}$ are respectively defined as 0 and $\infty$.

$$
\text { Furthermore, } \quad \operatorname{Pr}\left[\mathrm{SINR}^{\text {Link }}>\gamma \mid \text { LoS }\right] \text { and }
$$
$\operatorname{Pr}\left[\mathrm{SINR}^{\text {Link }}>\gamma \mid N L o S\right]$ are respectively computed by

$$
\begin{aligned}
& \operatorname{Pr}\left[\operatorname{SINR}^{L i n k}>\gamma \mid \operatorname{LoS}\right] \\
& \stackrel{(a)}{=} \exp \left(-\frac{\gamma P_{\mathrm{N}}^{L i n k}}{P^{L i n k} \zeta_{b_{o}}^{\mathrm{B} 2 \mathrm{U}}}\right) \mathscr{L}_{I_{\mathrm{agg}}^{L i n k, \mathrm{D}}}\left(\frac{\gamma}{P^{L i n k} \zeta_{b_{o}}^{\mathrm{B} 2 \mathrm{U}, \mathrm{L}}}\right) \\
& \times \mathscr{L}_{I_{\mathrm{agg}}^{\operatorname{Link} \mathrm{U}}}\left(\frac{\gamma}{P^{L i n k} \zeta_{b_{o}}^{\mathrm{B} 2 \mathrm{U}, \mathrm{L}}}\right),
\end{aligned}
$$

and

$$
\begin{aligned}
& \operatorname{Pr}\left[\mathrm{SINR}^{L i n k}>\gamma \mid \mathrm{NLoS}\right] \\
& =\exp \left(-\frac{\gamma P_{\mathrm{N}}^{L i n k}}{P^{L i n k} \zeta_{b_{o}}^{\mathrm{B} 2 \mathrm{U}}}\right) \mathscr{L}_{I_{\mathrm{agg}}^{L i n k, \mathrm{D}}}\left(\frac{\gamma}{P^{L i n k} \zeta_{b_{o}}^{\mathrm{B} 2 \mathrm{U}, \mathrm{NL}}}\right) \\
& \times \mathscr{L}_{I_{\mathrm{agg}}^{L i n k, \mathrm{U}}}\left(\frac{\gamma}{P^{L i n k} \zeta_{b_{o}}^{\mathrm{B} 2 \mathrm{U}, \mathrm{NL}}}\right),
\end{aligned}
$$

where $(a)$ is due to the independence of $R V I_{\mathrm{agg}}^{\operatorname{Link}, \mathrm{D}}$ and $I_{\text {agg }}^{\text {Link }, \mathrm{U}}$, moreover, $\mathscr{L}_{I_{\mathrm{agg}}^{\text {Link, }}}(s)$ and $\mathscr{L}_{I_{\mathrm{agg}}^{\text {Link, }}}(s)$ are the Laplace transform of $R V I_{\mathrm{agg}}^{\mathrm{Link}, \mathrm{D}}$ and $I_{\mathrm{agg}}^{\mathrm{Ling}, \mathrm{U}}$ evaluated at $s$ respectively.

Proof: See Appendix D.

Note that the DL-to-DL interference and the UL-to-UL interference of dense cellular networks have been studied in our previous papers [17], [23]. Different from [17] and [23], in this paper, we focus on the downlink and uplink performance analysis of dense small cell networks with dynamic TDD. Moreover, the novelties of the PHY layer analysis lie in the analysis of the inter-cell inter-link interference, i.e., the DLto-UL interference $I_{\mathrm{agg}}^{\mathrm{U}, \mathrm{D}}$ and the UL-to-DL interference $I_{\mathrm{agg}}^{\mathrm{D}, \mathrm{U}}$. The Laplace transforms of the inter-cell inter-link interference, i.e., $\mathscr{L}_{I_{\mathrm{agg}}^{\mathrm{U}, \mathrm{D}}}(s)$ and $\mathscr{L}_{I_{\mathrm{agg}}^{\mathrm{D}, \mathrm{U}}}(s)$, can be characterized by the following two Lemmas.

Lemma 7. The Laplace transform $\mathscr{L}_{I_{\mathrm{agg}}^{\mathrm{U}, \mathrm{D}}}(s)$ of the DL-to$U L$ interference $I_{\mathrm{agg}}^{\mathrm{U}, \mathrm{D}}$, i.e., the aggregate interference from $D L$ transmitting $B S$ to the UL typical BS, evaluated at $s$ can be computed as

$$
\mathscr{L}_{I_{\text {agg }}^{\mathrm{U}, \mathrm{D}}}(s)=\exp \left(-2 \pi p^{\mathrm{D}} \tilde{\lambda} \int_{r}^{\infty}\left[\frac{1}{1+\left(s P^{\mathrm{D}} \zeta^{D 2 U}\right)^{-1}}\right] x d x\right) .
$$


Proof: By assuming the distance between BSs $(x)$ are larger than the distance from the typical UE to the typical BS $(r),(31)$ is proved.

Lemma 8. The Laplace transform $\mathscr{L}_{I_{\mathrm{agg}}^{\mathrm{D}, \mathrm{U}}}(s)$ of the UL-to$D L$ interference $I_{\mathrm{agg}}^{\mathrm{D}, \mathrm{U}}$, i.e., the aggregate interference from $U L$ transmitting UE to the DL typical UE, evaluated at $s$ can be computed as

$$
\mathscr{L}_{I_{\mathrm{agg}}^{\mathrm{D}, \mathrm{U}}}(s)=\exp \left(-2 \pi p^{\mathrm{U}} \tilde{\lambda} \int_{r}^{\infty}\left[\frac{1}{1+\left(s P^{\mathrm{U}} \zeta^{U 2 U}\right)^{-1}}\right] x d x\right) .
$$

Proof: By assuming the locations of UEs are replaced by their serving BSs, and the distance between BSs $(x)$ are larger than the distance from the typical UE to the typical BS $(r)$, (32) is proved.

Note that the UL performance of dynamic TDD is important because the UL SINR is vulnerable to the DL-to-UL interference [22]. If the DL-to-UL interference is not properly treated, the UL coverage probability of dynamic TDD will suffer from a performance loss compared with static TDD. Therefore we propose a simple full interference cancellation (IC) scheme for the UL of dynamic TDD, which will be addressed in the following subsection.

\section{B. Coverage Probability with Interference Cancellation}

In this Subsection, we consider a simple full interference cancellation (IC) scheme to demonstrate the gain with interference mitigation. We assume that for the UL of dynamic TDD, all the DL transmitters with instantaneous interference power above $\delta$ can be cancelled at the typical BS.

Lemma 9. The Laplace transform with IC $\mathscr{L}_{I_{\mathrm{agg}}^{\mathrm{D}, \mathrm{U}}}(s)$ of $I_{\mathrm{agg}}^{\mathrm{D}, \mathrm{U}}$ evaluated at $s$ can be computed as

$$
\begin{aligned}
& \mathscr{L}_{I_{\mathrm{agg}}^{\mathrm{D}, \mathrm{U}}}(s) \\
& =\exp \left(-2 \pi p^{\mathrm{D}} \lambda \int_{0}^{d_{1}}\left(1-\mathbb{E}\left[I\left(h<\frac{\delta}{P^{\mathrm{B}} \zeta^{B 2 B, L}}\right)\right.\right.\right. \\
& \left.\left.\left.\times \exp \left(-s P^{\mathrm{B}} \zeta^{B 2 B, L} h\right)\right]\right)\left(1-\frac{x}{d_{1}}\right) x d x\right) \\
& \times \exp \left(-2 \pi p^{\mathrm{D}} \lambda \int_{0}^{d_{1}}\left(1-\mathbb{E}\left[I\left(h<\frac{\delta}{P^{\mathrm{B}} \zeta^{B 2 B, N L}}\right)\right.\right.\right. \\
& \left.\left.\left.\times \exp \left(-s P^{\mathrm{B}} \zeta^{B 2 B, N L} h\right)\right]\right)\left(\frac{x}{d_{1}}\right) x d x\right) \\
& \times \exp \left(-2 \pi p^{\mathrm{D}} \lambda \int_{d_{1}}^{\infty}\left(1-\mathbb{E}\left[1\left(h<\frac{\delta}{P^{\mathrm{B}} \zeta^{B 2 B, N L}}\right)\right.\right.\right. \\
& \left.\left.\left.\times \exp \left(-s P^{\mathrm{B}} \zeta^{B 2 B, N L} h\right)\right]\right) x d x\right),
\end{aligned}
$$

where the expectation over $h$ can be derived as

$$
\begin{aligned}
& \mathbb{E}\left[I\left(h<\frac{\delta}{P^{\mathrm{B}} \zeta^{B 2 B}}\right) \exp \left(-s P^{\mathrm{B}} \zeta^{B 2 B} h\right)\right] \\
& =\int_{0}^{\frac{P^{\mathrm{B}} \zeta^{B 2 B}}{2}} \exp \left(-s P^{\mathrm{B}} \zeta^{B 2 B} h\right) \exp (-h) d h \\
& =-\frac{1}{s P^{\mathrm{B}} \zeta^{B 2 B}+1}\left(\exp \left(-\frac{\delta\left(s P^{\mathrm{B}} \zeta^{B 2 B}+1\right)}{P^{\mathrm{B}} \zeta^{B 2 B}}\right)-1\right) .
\end{aligned}
$$

Proof: By assuming the fading $h$ of interference is smaller than $\frac{\delta}{P^{\mathrm{B}} \zeta^{\mathrm{B} 2 \mathrm{~B}}}$, Lemma 9 is proved.

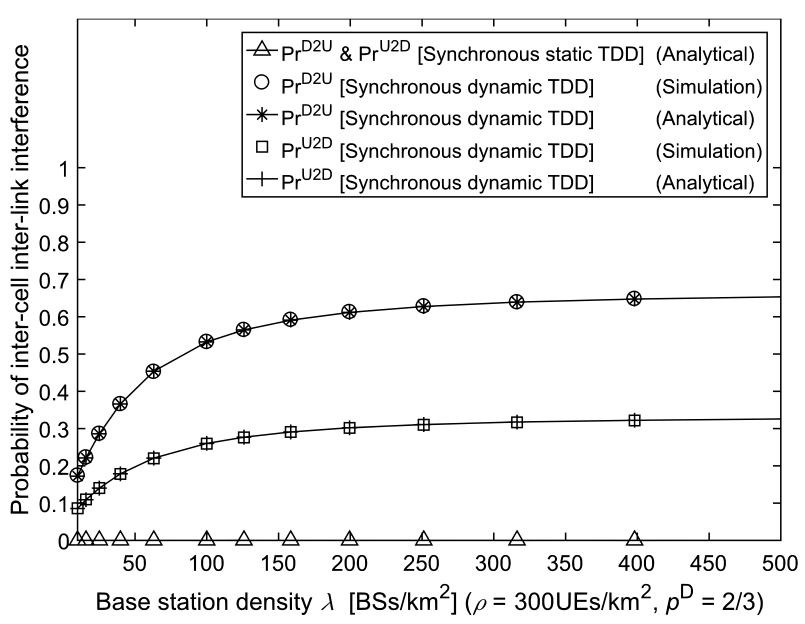

Fig. 3. The probability of inter-cell inter-link interference.

\section{Study of A 3GPP Special Case}

As a special case for Theorem 6, we consider a path loss function adopted in the 3GPP as [2]

$\zeta(r)= \begin{cases}A^{\mathrm{L}} r^{\alpha^{\mathrm{L}}}, & \text { LoS with probability } \operatorname{Pr}^{\mathrm{L}}(r) \\ A^{\mathrm{NL}} r^{\alpha^{\mathrm{NL}}}, & \text { NLoS with probability }\left(1-\operatorname{Pr}^{\mathrm{L}}(r)\right)\end{cases}$

together with a linear LoS probability function of $\mathrm{Pr}^{L}(r)$, defined in the 3GPP as [2]

$$
\operatorname{Pr}^{\mathrm{B} 2 \mathrm{U}, \mathrm{L}}(r)=\operatorname{Pr}^{\mathrm{B} 2 \mathrm{~B}, \mathrm{~L}}(r)= \begin{cases}1-\frac{r}{d_{1}}, & 0<r \leq d_{1} \\ 0, & r>d_{1}\end{cases}
$$

where $d_{1}$ is the cut-off distance of the LoS link.

In addition, a simple LoS probability function for $\operatorname{Pr}^{\mathrm{U} 2 \mathrm{U}, \mathrm{L}}(r)$ is given by [2]

$$
\operatorname{Pr}^{\mathrm{U} 2 \mathrm{U}, \mathrm{L}}(r)= \begin{cases}1, & 0<r \leq 50 \mathrm{~m} \\ 0, & r>50 \mathrm{~m}\end{cases}
$$

For the 3GPP special case, according to Theorem 6, $p^{\mathrm{cov}, \text { Link }}(\lambda, \gamma)$ can then be computed by

$$
p^{\mathrm{cov}, \text { Link }}(\lambda, \gamma)=\sum_{n=1}^{2}\left(T_{n}^{\mathrm{L}, \text { Link }}+T_{n}^{\mathrm{NL}, \text { Link }}\right) .
$$

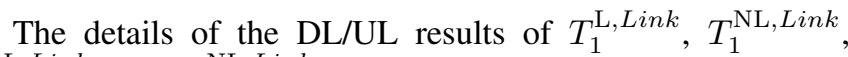
$T_{2}^{\mathrm{L}, \text { Link }}$, and $T_{2}^{\mathrm{NL}, \text { Link }}$ are investigated in Appendix $\mathrm{E}$ and Appendix $\mathrm{F}$ respectively.

\section{Simulation Results}

In this section, we present numerical results to validate the accuracy of our analysis. In our simulation, we adopt the following parameters recommended by the $3 \mathrm{GPP}$ [2]. In (3), $N=2$ and for $n \in\{1,2\}, A_{n}^{\mathrm{B} 2 \mathrm{U}, \mathrm{L}}=10^{-10.38}, A_{n}^{\mathrm{B} 2 \mathrm{U}, \mathrm{NL}}=$ $10^{-14.54}, A_{n}^{\mathrm{B} 2 \mathrm{~B}, \mathrm{~L}}=10^{-9.84}, A_{n}^{\mathrm{B} 2 \mathrm{~B}, \mathrm{NL}}=10^{-16.94}, A_{n}^{\mathrm{U} 2 \mathrm{U}, \mathrm{L}}=$ $10^{-9.85}, A_{n}^{\mathrm{U} 2 \mathrm{U}, \mathrm{NL}}=10^{-17.58}, \alpha_{n}^{\mathrm{B} 2 \mathrm{U}, \mathrm{L}}=2.09, \alpha_{n}^{\mathrm{B} 2 \mathrm{U}, \mathrm{NL}}=$ $3.75, \alpha_{n}^{\mathrm{B} 2 \mathrm{~B}, \mathrm{~L}}=2, \alpha_{n}^{\mathrm{B} 2 \mathrm{~B}, \mathrm{NL}}=4, \alpha_{n}^{\mathrm{U} 2 \mathrm{U}, \mathrm{L}}=2, \alpha_{n}^{\mathrm{U} 2 \mathrm{U}, \mathrm{NL}}=4$.

Besides, according to [2], the power values are set to: $P_{\mathrm{N}}^{\mathrm{D}}=-95 \mathrm{dBm}, P_{\mathrm{N}}^{\mathrm{U}}=-91 \mathrm{dBm}, P^{\mathrm{D}}=24 \mathrm{dBm}$, and $P^{\mathrm{U}}=23 \mathrm{dBm}$ [2]. In addition, the UE density is set to $\rho=300 \mathrm{UEs} / \mathrm{km}^{2}$, which leads to $q=4.05$ in (10) [14] 


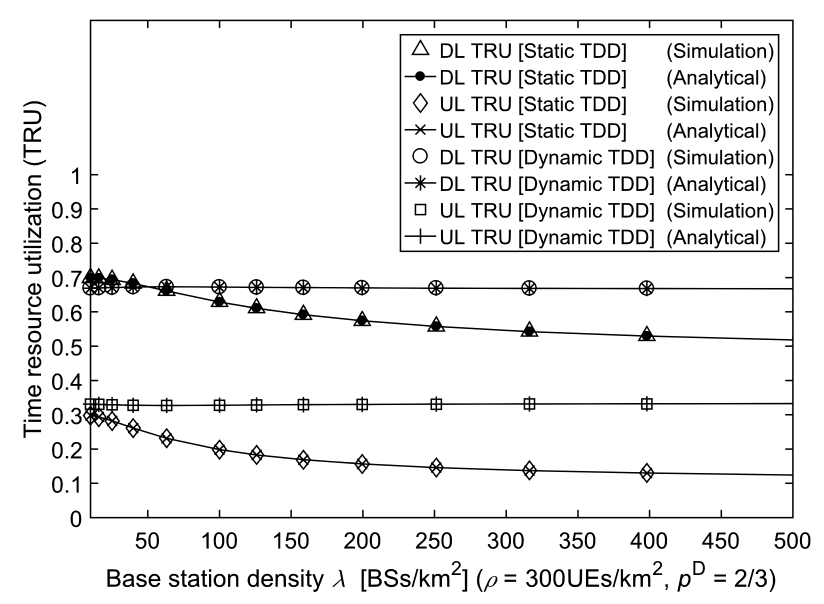

Fig. 4. The average DL/UL TRU $\kappa^{\mathrm{D}}$ and $\kappa^{\mathrm{U}}$.

Finally, we assume that $\gamma=1, p^{\mathrm{D}}=\frac{2}{3}$ and $T=10$. Thus, for static TDD, we have $N_{0}^{\mathrm{D}}=7$ and $N_{0}^{\mathrm{U}}=3$ in (22), which achieves the best match with $p^{\mathrm{D}}$ and $p^{\mathrm{U}}$, according to (16).

\section{A. Validation of the Results on the Probabilities of Inter-Cell Inter-Link Interference}

The analytical and simulation results of $\operatorname{Pr}^{\mathrm{D} 2 \mathrm{U}}$ and $\operatorname{Pr}^{\mathrm{U}} 2 \mathrm{D}$ are plotted in Fig. 3. From this figure, we can draw the following observations:

- The analytical and simulation results match well, which validates the accuracy of our analysis.

- For static TDD, $\operatorname{Pr}^{\mathrm{D} 2 \mathrm{U}}$ and $\operatorname{Pr}^{\mathrm{U} 2 \mathrm{D}}$ are zeros.

- For dynamic TDD in the synchronous case, as the BS density $\lambda$ increases, $\operatorname{Pr}^{\mathrm{D} 2 \mathrm{U}}$ and $\operatorname{Pr}^{\mathrm{U} 2 \mathrm{D}}$ gradually grow and converge to the results of dynamic TDD in the asynchronous case. This is because:

- when $\lambda$ increases, the UE number in each active BS decreases; and

- hence, when $\lambda$ is high enough to reach the limit of one UE per active BS, all the subframes will be used as either DL ones or UL ones, the probability of which solely depends on $p^{\mathrm{D}}$ or $p^{\mathrm{U}}$.

\section{B. Validation of the Results on the Time Resource Utilization}

The analytical and simulation results of $\kappa^{\mathrm{D}}$ and $\kappa^{\mathrm{U}}$ are plotted in Fig. 4. From this figure, we can see that:

- For static TDD, $\kappa^{\mathrm{D}}$ starts from 0.7 and decreases as $\lambda$ increases. This is because $N_{0}^{\mathrm{D}}=7$ and $T=10$, and thus static TDD maintains $N_{0}^{\mathrm{U}}=3 \mathrm{UL}$ subframes even if there is no $\mathrm{UL}$ data request in a $\mathrm{BS}$, leading to a large time resource waste. Moreover, the sum of $\kappa^{\mathrm{D}}$ and $\kappa^{\mathrm{U}}$ is much less than one when $\lambda$ is large, e.g., $\lambda=500 \mathrm{BSs} / \mathrm{km}^{2}$, showing the inefficiency of static TDD in dense SCNs.

- For dynamic TDD, $\kappa^{\mathrm{D}}$ converges to $p^{\mathrm{D}}=\frac{2}{3}$ as $\lambda$ increases. This is because when $\lambda$ is high enough to reach the limit of one UE per active BS, all the subframes will be used as DL ones with a probability of $p^{\mathrm{D}}$. Moreover, the sum of $\kappa^{\mathrm{D}}$ and $\kappa^{\mathrm{U}}$ always equals to one, thanks to the dynamic adaption of DL/UL subframes to DL/UL data requests.

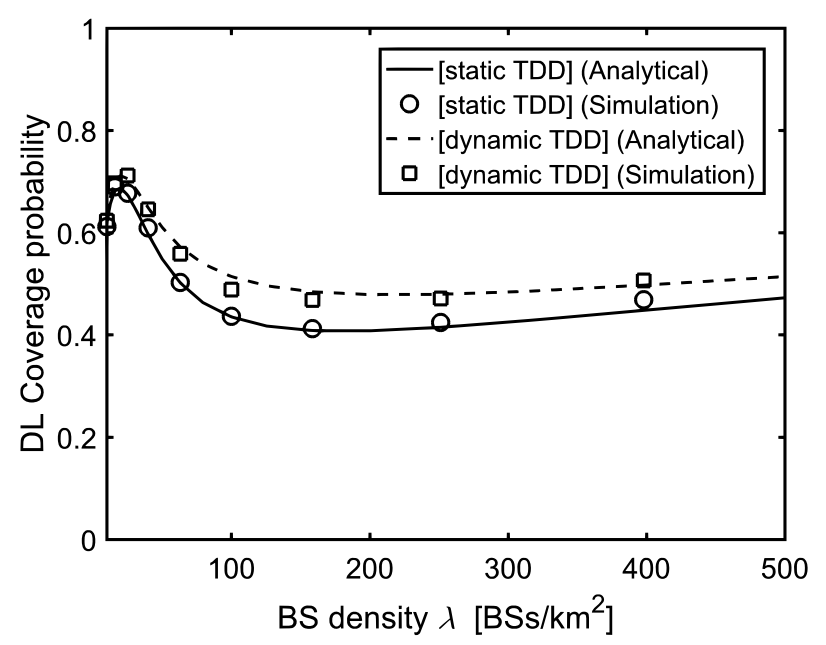

Fig. 5. The DL coverage probability $p^{\mathrm{cov}, \mathrm{D}}(\lambda, \gamma)$ vs. the BS density $\lambda$ with SINR threshold $\gamma=0 \mathrm{~dB}$.

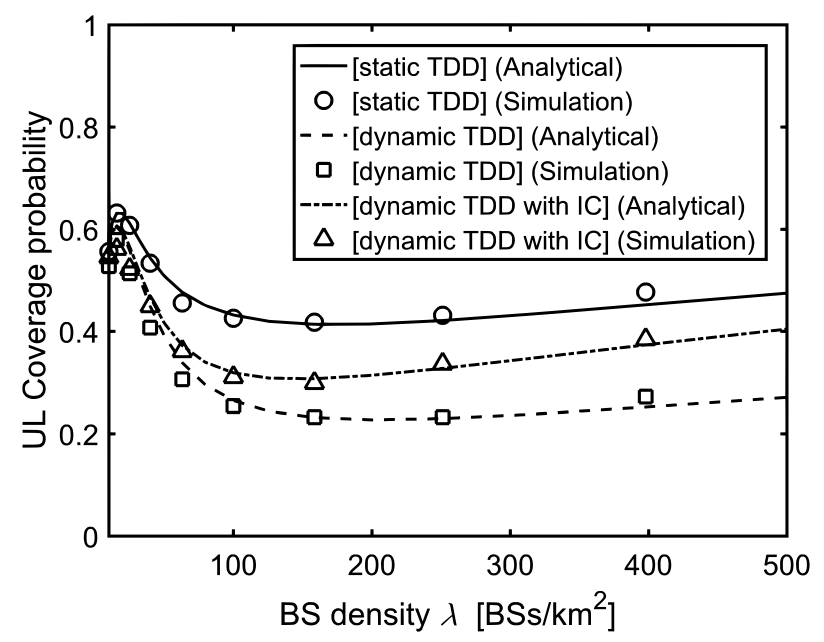

Fig. 6. The UL coverage probability $p^{\operatorname{cov}, \mathrm{U}}(\lambda, \gamma)$ vs. the BS density $\lambda$ with SINR threshold $\gamma=0 \mathrm{~dB}$.

\section{Validation of the Analytical Results of $p^{\operatorname{cov}, \text { Link }}(\lambda, \gamma)$}

In the case of the linear 3GPP path loss model as proposed in Subsection $\mathrm{V}-\mathrm{C}$, the results of $p^{\mathrm{cov}, \mathrm{D}}(\lambda, \gamma)$ and $p^{\mathrm{cov}, \mathrm{U}}(\lambda, \gamma)$ against the BS density for $\gamma=0 \mathrm{~dB}$ are plotted in Fig. 5 and Fig. 6 respectively. Note that our analytical results on $\operatorname{Pr}^{\mathrm{D} 2 \mathrm{U}}$ and $\operatorname{Pr}^{\mathrm{U} 2 \mathrm{D}}$ are used for the simulation of $I_{\mathrm{agg}}^{\mathrm{D}}$ and $I_{\mathrm{agg}}^{\mathrm{U}}$ in (7). As can be observed from Fig. 5 and Fig. 6, our analytical results with the assumptions in Lemma 7 and Lemma 8 match the simulation results well, and thus we will only use analytical results of $p^{\text {cov, Link }}(\lambda, \gamma)$ in our discussion hereafter.

From Fig. 5, we can observe that:

- For static TDD, when considering both LoS and NLoS transmissions, the DL coverage probability shows a complicated performance trend. The details are described as follows:

- When the SCN is sparse and thus noise-limited, the DL coverage probability of static TDD given by the proposed analysis grows as $\lambda$ increases.

- However, when the network is dense enough, the DL coverage probability decreases as $\lambda$ increases, due to 
the transition of a large number of interference paths from NLoS to LoS. Particularly, during this region, interference increases at a faster rate than the signal due to the transition from mostly NLoS interference to $\operatorname{LoS}$ interference, thereby causing a drop in the SINR hence the coverage probability.

- In more detail, the coverage probability given by the proposed analysis peaks at a certain density $\lambda_{0}$. When $\lambda$ is further increased far above $\lambda_{0}$, the coverage probability decreases at a slower pace because both the signal power and the interference power are LoS dominated and increase at approximately the same rate. There are still more and more interferers whose signal reach the typical BS via LoS paths but their effect is smaller than the dominating interferers.

- At last, the coverage probability picks up the increasing speed because the number of interferers is limited by the UE density $\rho$.

- For dynamic TDD, the DL coverage probability presents a similar behavior compared to the static TDD. Note that the DL coverage probability of dynamic TDD performs better than the static TDD, due to part of the strong DL interference becomes weak UL interference in the scenario of DL of dynamic TDD.

From Fig. 6, we can observe that:

- On one hand, when considering both LoS and NLoS transmissions, the UL coverage probability of dynamic TDD presents a similar behavior compared to the static TDD, due to the similar reason as the DL.

- On the other hand, the UL coverage probability of dynamic TDD performs worse than the static TDD, due to part of the weak UL interference becomes strong DL interference in the scenario of UL of dynamic TDD. Therefore it is necessary to apply the interference cancellation (IC) scheme to the UL of dynamic TDD. With the IC proposed in subsec V-B, the UL coverage probability of dynamic TDD is enhanced, although still poorer than the static TDD.

However, from Fig. 6, we should not conclude that for the UL dynamic TDD exhibits no significant performance gain compared with static TDD due to the detrimental DLto-UL interference. Instead, we need to investigate the ASE performance, which includes the time resource utilization and is shown in the following Subsection.

\section{The ASE Performance}

From Fig. 7, we can conclude that:

- For static TDD, the DL ASE also shows a complicated performance trend. The details are described as follows:

- When the SCN is sparse and thus noise-limited, the DL ASE of static TDD given by the proposed analysis grows as $\lambda$ increases.

- However, when the network is dense enough, the DL ASE decreases as $\lambda$ increases, due to the decrease of coverage probability and TRU.

- When $\lambda$ is further increased, the DL ASE picks up the increasing speed, because the increasing effect

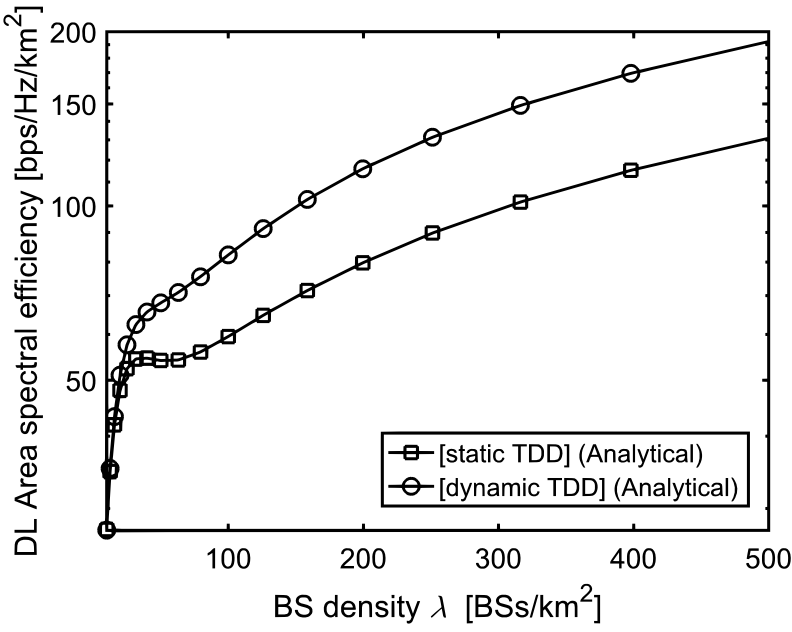

Fig. 7. DL area spectral efficiency $A S E^{\mathrm{D}}\left(\lambda, \gamma_{0}\right)$ vs. the BS density $\lambda$ with SINR threshold $\gamma_{0}=0 \mathrm{~dB}$.

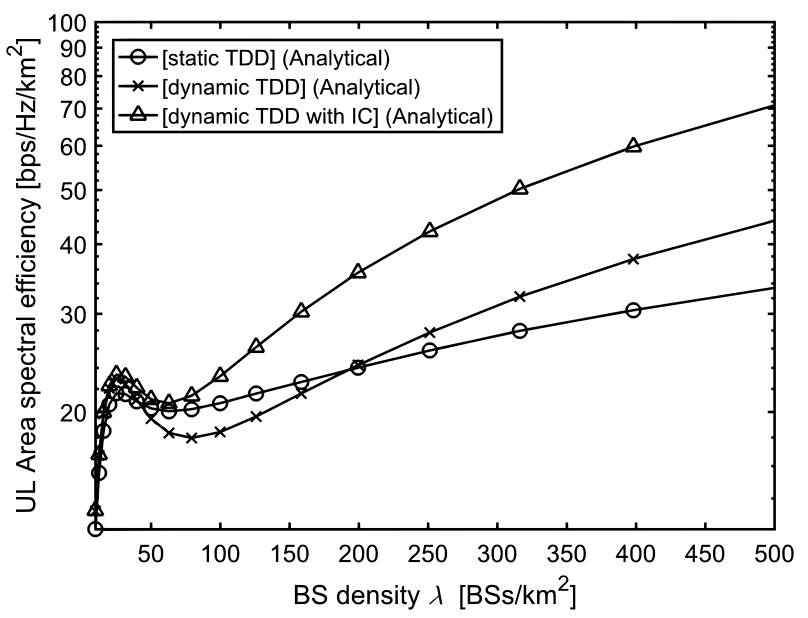

Fig. 8. UL area spectral efficiency $A S E^{\mathrm{U}}\left(\lambda, \gamma_{0}\right)$ vs. the BS density $\lambda$ with SINR threshold $\gamma_{0}=0 \mathrm{~dB}$.

of active BS density and coverage probability is stronger than the decreasing effect of TRU.

- For dynamic TDD, the details of the behavior of the DL ASE are described as follows:

- When the SCN is sparse and thus noise-limited, the DL ASE grows as $\lambda$ increases. When the network is dense enough, the DL ASE increases at a slower rate as $\lambda$ increases, due to the decrease of coverage probability. Note that the effect of the decrease of coverage probability is weaker than the increase of active BS density, as a result, the DL ASE of dynamic TDD keeps increasing and doesn't present decrease. When $\lambda$ is further increased, the DL ASE slightly picks up the increasing speed and increases almost linearly as $\lambda$ increases.

- The DL ASE exhibits performance gain compared with static TDD due to the beneficial UL-to-DL interference and the TRU gain, especially for $5 \mathrm{G}$ dense SCNs [1], i.e., $\lambda>100 \mathrm{BSs} / \mathrm{km}^{2}$.

From Fig. 8, we can conclude that: 
- The UL ASE of dynamic TDD presents a similar behavior compared to static TDD, due to the similar reason as the DL.

- The UL performance is important because the UL SINR is vulnerable to the DL-to-UL interference [22]. If the DL-to-UL interference is not properly treated, the UL ASE of dynamic TDD will suffer from a performance loss compared with static TDD, for the BS density range of $30<\lambda<200 \mathrm{BSs} / \mathrm{km}^{2}$.

- To mitigate such interference, we investigate the effectiveness of the full interference cancellation (IC), which removes all DL-to-UL interfering signals based on instantaneous DL-to-UL interference power. As discussed in Subsection V-B, the IC threshold is set as $\delta=-50$ $\mathrm{dBm}$. Note that the results of full IC, i.e., cancelling a subset of interferers, would be ideal. The analysis of other interference mitigation methods, e.g., clustering, UL power boosting, etc., is left as possible future work.

- Dynamic TDD with IC can achieve larger ASE in the UL compared with static TDD, mainly due to the dynamic adaption of DL/UL subframes to DL/UL data requests. The performance gain of dynamic TDD with IC in the UL is larger as the BS density $\lambda$ increases. When $\lambda=$ $500 \mathrm{BSs} / \mathrm{km}^{2}$, the UL ASE of static TDD and that of dynamic TDD with full IC are around $30 \mathrm{bps} / \mathrm{Hz} / \mathrm{km}^{2}$ and $70 \mathrm{bps} / \mathrm{Hz} / \mathrm{km}^{2}$, respectively.

\section{CONCLUSION}

In this paper, we have investigated the impact of synchronous dynamic TDD in the performance of the DL/UL of dense SCNs. Analytical results are obtained for the DL/UL TRU, coverage probability and ASE. Specifically, we find that

- The DL/UL TRU varies across TDD subframes, and that of dynamic TDD can achieve an increasingly higher average total TRU than static TDD with the network densification of up to $75.4 \%$.

- With the beneficial UL-to-DL interference and MAC layer gain, dynamic TDD can achieve an increasingly higher average DL ASE than static TDD with the network densification of up to $50 \%$.

- With the MAC layer gain and proper IC, dynamic TDD can achieve an increasingly higher average UL ASE than static TDD with the network densification of up to $100 \%$.

As our future work, we will consider other factors of realistic networks in the theoretical analysis for SCNs, such as the introduction of Rician fading or Nakagami fading, because the multi-path fading model is also affected by the LoS and NLoS transmissions.

\section{Appendix A: Proof of TheOrem 1}

Based on (17) and conditioned on $\tilde{K}=\tilde{k}$, the probability of performing a DL transmission in subframe $l$ $(l \in\{1,2, \ldots, T\})$ can be calculated as

$$
\begin{aligned}
q_{l, \tilde{k}}^{\mathrm{D}} & =\operatorname{Pr}\left[Y_{l}={ }^{\prime} \mathrm{D}^{\prime} \mid \tilde{K}=\tilde{k}\right] \\
& \stackrel{(a)}{=} \operatorname{Pr}\left[N^{\mathrm{D}} \geq l\right]=1-F_{N^{\mathrm{D}}}(l-1),
\end{aligned}
$$

where $Y_{l}$ denotes the link direction for the transmission on the $l$-th subframe, which takes a string value of ' $D$ ' and ' $U$ ' for the DL and the UL, respectively. Besides, the step $(a)$ of (39) is due to the LTE TDD configuration structure shown in Fig. 2, and $F_{N^{\mathrm{D}}}\left(n^{\mathrm{D}}\right)$ is the cumulative mass function (CMF) of $N^{\mathrm{D}}$ in an active BS, which is written as

$$
F_{N^{\mathrm{D}}}\left(n^{\mathrm{D}}\right)=\operatorname{Pr}\left[N^{\mathrm{D}} \leq n^{\mathrm{D}}\right]=\sum_{i=0}^{n^{\mathrm{D}}} f_{N^{\mathrm{D}}}(i) .
$$

Considering the dynamic allocation of subframe to the DL and the UL in dynamic TDD, the conditional probability of performing an UL transmission in subframe $l$ is given by

$$
q_{l, \tilde{k}}^{\mathrm{U}}=1-q_{l, \tilde{k}}^{\mathrm{D}}=F_{N^{\mathrm{D}}}(l-1) .
$$

Furthermore, the unconditional probabilities of performing a DL and UL transmissions on the $l$-th subframe, i.e., $q_{l}^{\mathrm{D}}$ and $q_{l}^{\mathrm{U}}$, can be respectively derived by calculating the expected values of $q_{l, \tilde{k}}^{\mathrm{D}}$ and $q_{l, \tilde{k}}^{\mathrm{U}}$ over all the possible values of $\tilde{k}$ as shown in (19), which concludes our proof.

\section{APPENDiX B: ProOF OF THEOREM 2}

By examining the inter-cell inter-link interference for the $l$-th subframe $(l \in\{1,2, \ldots, T\})$ one by one, $\operatorname{Pr}^{\mathrm{D} 2 \mathrm{U}}$ can be derived as

$$
\begin{aligned}
\operatorname{Pr}^{\mathrm{D} 2 \mathrm{U}} & =\operatorname{Pr}\left[Z={ }^{\prime} \mathrm{D}^{\prime} \mid S={ }^{\prime} \mathrm{U}^{\prime}\right] \\
& =\sum_{l=1}^{T} \operatorname{Pr}\left[\left(Z={ }^{\prime} \mathrm{D}^{\prime} \mid L=l\right) \mid S={ }^{\prime} \mathrm{U}^{\prime}\right] \\
& \times \operatorname{Pr}\left[L=l \mid S={ }^{\prime} \mathrm{U}^{\prime}\right] \\
& \stackrel{(a)}{=} \sum_{l=1}^{T} q_{l}^{\mathrm{D}} \operatorname{Pr}\left[L=l \mid S={ }^{\prime} \mathrm{U}^{\prime}\right] \\
& \stackrel{(b)}{=} \sum_{l=1}^{T} q_{l}^{\mathrm{D}} \frac{\operatorname{Pr}\left[S={ }^{\prime} \mathrm{U}^{\prime} \mid L=l\right] \operatorname{Pr}[L=l]}{\operatorname{Pr}\left[S={ }^{\prime} \mathrm{U}^{\prime}\right]} \\
& \stackrel{(c)}{=} \sum_{l=1}^{T} q_{l}^{\mathrm{D}} \frac{q_{l}^{\mathrm{U}} \frac{1}{T}}{\frac{1}{T} \sum_{j=1}^{T} q_{j}^{\mathrm{U}}},
\end{aligned}
$$

where (a) is obtained from

$\operatorname{Pr}\left[\left(Z={ }^{\prime} \mathrm{D}\right.\right.$ ' $\left.\left.\mid L=l\right) \mid S={ }^{\prime} \mathrm{U}^{\prime}\right]=\operatorname{Pr}\left[Z={ }^{\prime} \mathrm{D}\right.$ ' $\left.\mid L=l\right]=q_{l}^{\mathrm{D}}$,

due to the independence of the events $\left(Z={ }^{\prime} \mathrm{D}^{\prime} \mid L=l\right)$ and ( $S=$ 'U'); (b) is valid because of the Bayes' Theorem; and (c) comes from the calculation on the probability of the signal being an UL one, which can be written by

$$
\begin{aligned}
\operatorname{Pr}\left[S={ }^{\prime} \mathrm{U}^{\prime}\right] & =\sum_{j=1}^{T} \operatorname{Pr}\left[S={ }^{\prime} \mathrm{U}^{\prime} \mid L=j\right] \operatorname{Pr}[L=j] \\
& =\frac{1}{T} \sum_{j=1}^{T} q_{j}^{\mathrm{U}} .
\end{aligned}
$$

Similarly, it is easy to derive the results for $\mathrm{Pr}^{\mathrm{U} 2 \mathrm{D}}$, which concludes our proof. 


\section{APPENDix C: PROOF OF THEOREM 4}

In static TDD, for a given UE number $\tilde{k}$ in an active $\mathrm{BS}$, the probabilities that no UE requests any DL data and no UE requests any UL data can be calculated by $f_{M^{\mathrm{D}}}(0)$ from (14) and $f_{M^{\mathrm{U}}}(0)$ from (15), respectively. Even in such cases, static TDD still unwisely allocates $N_{0}^{\mathrm{D}}$ and $N_{0}^{\mathrm{U}}$ subframes for the DL and the UL, respectively, which causes resource waste. The probabilities of such resource waste for the DL and the $\mathrm{UL}$ are denoted by $w^{\mathrm{D}}$ and $w^{\mathrm{U}}$, and they can be calculated as

$$
\left\{\begin{array}{l}
w^{\mathrm{D}}=\sum_{\tilde{k}=1}^{+\infty} f_{M^{\mathrm{D}}}(0) f_{\tilde{K}}(\tilde{k})=\sum_{\tilde{k}=1}^{+\infty}\left(1-p^{\mathrm{D}}\right)^{\tilde{k}} f_{\tilde{K}}(\tilde{k}) \\
w^{\mathrm{U}}=\sum_{\tilde{k}=1}^{+\infty} f_{M^{\mathrm{U}}}(0) f_{\tilde{K}}(\tilde{k})=\sum_{\tilde{k}=1}^{+\infty}\left(1-p^{\mathrm{U}}\right)^{\tilde{k}} f_{\tilde{K}}(\tilde{k})
\end{array} .\right.
$$

Excluding such resource waste from $N_{0}^{\mathrm{D}}$ and $N_{0}^{\mathrm{U}}$, we can obtain $\kappa^{\mathrm{D}}$ and $\kappa^{\mathrm{U}}$ for static TDD as

$$
\left\{\begin{array}{l}
\kappa^{\mathrm{D}}=\left(1-w^{\mathrm{D}}\right) \frac{N_{0}^{\mathrm{D}}}{T} \\
\kappa^{\mathrm{U}}=\left(1-w^{\mathrm{U}}\right) \frac{N_{0}^{\mathrm{U}}}{T}
\end{array} .\right.
$$

Our proof is thus completed by plugging (44), (14) and (15) into (45), followed by computing $\kappa$ from (5).

\section{Appendix D: Proof of TheOREM 6}

The proof of $p^{\text {cov }, \text { Link }}(\lambda, \gamma), f_{R, n}^{\mathrm{L}}(r)$ and $f_{R, n}^{\mathrm{NL}}(r)$ are given in our previous papers [17], [23]. Therefore we move on to evaluate $\operatorname{Pr}\left[\mathrm{SINR}^{\text {Link }}>\gamma \mid \mathrm{LoS}\right]$ in (29) as

$$
\begin{aligned}
& \operatorname{Pr}\left[\operatorname{SINR}^{\text {Link }}>\gamma \mid \operatorname{LoS}\right] \\
& =\operatorname{Pr}\left[h>\frac{\gamma\left(I_{\mathrm{agg}}^{L i n k, \mathrm{D}}+I_{\mathrm{agg}}^{L i n k, \mathrm{U}}+P_{\mathrm{N}}^{L i n k}\right)}{P^{L i n k} \zeta_{b_{o}}^{\mathrm{B} 2 \mathrm{U}}(r)} \mid \operatorname{LoS}\right] \\
& =\mathbb{E}_{\left(I_{\text {agg }}^{L i n k, \mathrm{D}}+I_{\text {agg }}^{L i n k, \mathrm{U}}\right)}\left\{\exp \left(-\frac{\gamma\left(I_{\text {agg }}^{L i n k, \mathrm{D}}+I_{\text {agg }}^{L i n k, \mathrm{U}}+P_{\mathrm{N}}^{L i n k}\right)}{P^{L i n k} \zeta_{b_{o}}^{\mathrm{B} 2 \mathrm{U}}(r)}\right)\right\} \\
& =\exp \left(-\frac{\gamma P_{\mathrm{N}}^{L i n k}}{P^{L i n k} \zeta_{b_{o}}^{\mathrm{B} 2 \mathrm{U}}(r)}\right) \\
& \times \mathbb{E}_{\left(I_{\text {agg }}^{L i n k, \mathrm{D}}+I_{\text {agg }}^{L i n k, \mathrm{U}}\right)}\left\{\exp \left(-\frac{\gamma\left(I_{\text {agg }}^{L i n k, \mathrm{D}}+I_{\text {agg }}^{L i n k, \mathrm{U}}\right)}{P^{L i n k} \zeta_{b_{o}}^{\mathrm{B} U}(r)}\right)\right\} \\
& =\exp \left(-\frac{\gamma P_{\mathrm{N}}^{L i n k}}{P^{L i n k} \zeta_{b_{o}}^{\mathrm{B2O}}(r)}\right) \mathscr{L}_{I_{\mathrm{agg}}^{L i n k, \mathrm{D}}}\left(\frac{\gamma\left(I_{\mathrm{agg}}^{\operatorname{Link}, \mathrm{D}}\right)}{P^{L i n k} \zeta_{b_{o}}^{\mathrm{BLO}}(r)}\right) \\
& \times \mathscr{L}_{I_{\mathrm{agg}}^{\mathrm{Link}, \mathrm{U}}}\left(\frac{\gamma\left(I_{\mathrm{agg}}^{\mathrm{Link}, \mathrm{U}}\right)}{P^{\operatorname{Link} \zeta_{b_{o}}^{\mathrm{BLO}}(r)}}\right) .
\end{aligned}
$$

$\operatorname{Pr}\left[\mathrm{SINR}^{\text {Link }}>\gamma \mid \mathrm{NLoS}\right]$ can be derived in a similar way.

\section{Appendix E: DL Results of Subsection V-C}

For the DL, the coverage probability for the typical UE can be formulated as follows.

$T_{1}^{\mathrm{L}, \mathrm{D}}$ of $D L$

Lemma 10. The result of $T_{1}^{\mathrm{L}, \mathrm{D}}$ is the $D L$ coverage probability when the typical UE is associated with the typical BS with a LoS link of distance less than $d_{1}$. From Theorem $6, T_{1}^{\mathrm{L}, \mathrm{D}}$ can be derived as

$$
\begin{aligned}
& T_{1}^{\mathrm{L}, \mathrm{D}}=\int_{0}^{d_{1}} \exp \left(-\frac{\gamma P_{\mathrm{N}}^{\mathrm{D}}}{P^{\mathrm{D}} \zeta_{b_{o}}^{\mathrm{B} 2 \mathrm{~V}}}\right) \mathscr{L}_{I_{\mathrm{agg}}^{\mathrm{D}, \mathrm{D}}}\left(\frac{\gamma}{P^{\mathrm{D}} \zeta_{b_{o}}^{\mathrm{B2U}, \mathrm{L}}}\right) \\
& \times \mathscr{L}_{I_{\mathrm{agg}}^{\mathrm{D}, \mathrm{U}}}\left(\frac{\gamma}{P^{\mathrm{D}} \zeta_{b_{o}}^{\mathrm{BV} U, \mathrm{~L}}}\right) f_{R, 1}^{\mathrm{L}}(r) d r .
\end{aligned}
$$

The Laplace transform $\mathscr{L}_{I_{\mathrm{agg}}^{\mathrm{D}, \mathrm{U}}}(s)$ of $I_{\mathrm{agg}}^{\mathrm{D}, \mathrm{U}}$ evaluated at $s=$ $\frac{\gamma}{P^{\mathrm{D}} \zeta_{b_{o}}^{\mathrm{B} 2 \mathrm{U}, \mathrm{L}}}$ can be computed as

$$
\begin{aligned}
& \mathscr{L}_{I_{\mathrm{agg}}^{\mathrm{D}, \mathrm{U}}}(s)=\exp \left(-2 \pi p^{\mathrm{U}} \lambda\left(\int_{r_{1}}^{50}\left[\frac{1}{1+\left(s P^{\mathrm{U}} \zeta^{U 2 U, L}\right)^{-1}}\right] x d x\right.\right. \\
& \left.\left.+\int_{50}^{\infty}\left[\frac{1}{1+\left(s P^{\mathrm{U}} \zeta^{U 2 U, N L}\right)^{-1}}\right] x d x\right)\right) .
\end{aligned}
$$

$T_{1}^{\mathrm{NL}, \mathrm{D}}$ of $D L$

Lemma 11. The DL coverage probability when $0<r \leq d_{1}$ and the signal is NLoS can be derived as

$$
\begin{aligned}
& T_{1}^{\mathrm{NL}, \mathrm{D}}=\int_{0}^{d_{1}} \exp \left(-\frac{\gamma P_{\mathrm{N}}^{\mathrm{D}}}{P^{\mathrm{D}} \zeta_{b_{o}}^{\mathrm{B} 2 \mathrm{U}}}\right) \mathscr{L}_{I_{\mathrm{agg}}^{\mathrm{D}, \mathrm{D}}}\left(\frac{\gamma}{P^{\mathrm{D}} \zeta_{b_{o}}^{\mathrm{B} 2 \mathrm{NL}}}\right) \\
& \times \mathscr{L}_{I_{\mathrm{agg}}^{\mathrm{D}, \mathrm{U}}}\left(\frac{\gamma}{P^{\mathrm{D}} \zeta_{b_{o}}^{\mathrm{B2}, \mathrm{NL}}}\right) f_{R, 1}^{\mathrm{NL}}(r) d r,
\end{aligned}
$$

where the Laplace transform $\mathscr{L}_{I_{\mathrm{agg}}^{\mathrm{D}, \mathrm{U}}}(s)$ evaluated at $s=$ $\frac{\gamma}{P^{\mathrm{D}} \zeta_{b_{o}}^{\mathrm{BQU}, \mathrm{NL}}}$ can be derived as (48).

$T_{2}^{\mathrm{L}, \mathrm{D}}$ of $D L$

The result of $T_{2}^{\mathrm{L}, \mathrm{D}}$ is the DL coverage probability when the typical UE is associated with the typical BS with a LoS link of distance larger than $d_{1}$. From Theorem $6, T_{2}^{\mathrm{L}, \mathrm{D}}$ can be derived as

$$
T_{2}^{\mathrm{L}, \mathrm{D}}=\int_{d_{1}}^{\infty} \operatorname{Pr}\left[\mathrm{SINR}^{\text {Link }}>\gamma \mid r, \mathrm{LoS}\right] f_{R, 2}^{\mathrm{L}}(r) d r .
$$

Plugging $f_{R, 2}^{\mathrm{L}}(r)=0$ into (50), yields

$$
T_{2}^{\mathrm{L}, \mathrm{D}}=0 .
$$

$T_{2}^{\mathrm{NL}, \mathrm{D}}$ of $D L$

Lemma 12. The DL coverage probability when $r>d_{1}$ and the signal is NLoS can be derived as

$$
\begin{aligned}
& T_{2}^{\mathrm{NL}, \mathrm{D}}=\int_{d_{1}}^{\infty} \exp \left(-\frac{\gamma P_{\mathrm{N}}^{\mathrm{D}}}{P^{\mathrm{D}} \zeta_{b_{o}}^{\mathrm{B} 2 \mathrm{U}}}\right) \mathscr{L}_{I_{\mathrm{agg}}^{\mathrm{D}, \mathrm{D}}}\left(\frac{\gamma}{P^{\mathrm{D}} \zeta_{b_{o}}^{\mathrm{B} 2 \mathrm{~N}, \mathrm{NL}}}\right) \\
& \times \mathscr{L}_{I_{\mathrm{agg}}^{\mathrm{D}, \mathrm{U}}}\left(\frac{\gamma}{P^{\mathrm{D}} \zeta_{b_{o}}^{\mathrm{B} 2 \mathrm{~N}, \mathrm{NL}}}\right) f_{R, 2}^{\mathrm{NL}}(r) d r,
\end{aligned}
$$

and the Laplace transform $\mathscr{L}_{I_{\mathrm{agg}}^{\mathrm{D}, \mathrm{U}}}(s)$ of $I_{\mathrm{agg}}^{\mathrm{D}, \mathrm{U}}$ evaluated at $s=\frac{\gamma}{P^{\mathrm{D}} \zeta_{b_{o}}^{\mathrm{B2U}, \mathrm{NL}}}$ can be computed as

$$
\mathscr{L}_{I_{\mathrm{agg}}^{\mathrm{D}, \mathrm{U}}}(s)=\exp \left(-2 \pi p^{\mathrm{U}} \lambda \int_{r}^{\infty}\left[\frac{1}{1+\left(s P^{\mathrm{U}} \zeta^{\mathrm{U} 2 U, N L}\right)^{-1}}\right] x d x\right) .
$$

\section{Appendix F: UL Results of Subsection V-C}

For the UL, the coverage probability for the typical BS can be formulated as follows.

$T_{1}^{\mathrm{L}, \mathrm{U}}$ of $U L$

Lemma 13. The UL coverage probability when $0<r \leq d_{1}$ and the signal is LoS can be derived as

$$
\begin{aligned}
& T_{1}^{\mathrm{L}, \mathrm{U}}=\int_{0}^{d_{1}} \exp \left(-\frac{\gamma P_{\mathrm{N}}^{\mathrm{U}}}{P^{\mathrm{D}} \zeta_{b_{o}}^{\mathrm{B} 2 \mathrm{U}}}\right) \mathscr{L}_{I_{\mathrm{agg}}^{\mathrm{Ug}, \mathrm{D}}}\left(\frac{\gamma}{P^{\mathrm{U}} \zeta_{b_{o}}^{\mathrm{B} 2 \mathrm{~L}, \mathrm{~L}}}\right) \\
& \times \mathscr{L}_{I_{\mathrm{agg}}^{\mathrm{U}, \mathrm{U}}}\left(\frac{\gamma}{P^{\mathrm{U}} \zeta_{b_{o}}^{\mathrm{B2}}}\right) f_{R, 1}^{\mathrm{L}}(r) d r,
\end{aligned}
$$


and the Laplace transform $\mathscr{L}_{I_{\mathrm{agg}}^{\mathrm{U}, \mathrm{D}}}(s)$ of $I_{\mathrm{agg}}^{\mathrm{U}, \mathrm{D}}$ evaluated at $s=\frac{\gamma}{P^{\mathrm{U}} \zeta_{b_{o}}^{\mathrm{B} 2 \mathrm{U}, \mathrm{L}}}$ can be computed as

$$
\begin{aligned}
\mathscr{L}_{I_{\mathrm{agg}}^{\mathrm{U}, \mathrm{D}}}(s)= & \exp \left(-2 \pi p^{\mathrm{D}} \lambda \int_{0}^{d_{1}}\left[\frac{1}{1+\left(s P^{\mathrm{D}} \zeta^{B 2 B, L}\right)^{-1}}\right]\left(1-\frac{x}{d_{1}}\right) x d x\right) \\
& \times \exp \left(-2 \pi p^{\mathrm{D}} \lambda \int_{0}^{d_{1}}\left[\frac{1}{1+\left(s P^{\mathrm{D}} \zeta^{B 2 B, N L}\right)^{-1}}\right]\left(\frac{x}{d_{1}}\right) x d x\right) \\
& \times \exp \left(-2 \pi p^{\mathrm{D}} \lambda \int_{d_{1}}^{\infty}\left[\frac{1}{1+\left(s P^{\mathrm{D}} \zeta^{B 2 B, N L}\right)^{-1}}\right] x d x\right) .
\end{aligned}
$$

$T_{1}^{\mathrm{NL}, \mathrm{U}}$ of $U L$

Lemma 14. The UL coverage probability when $0<r \leq d_{1}$ and the signal is NLoS can be derived as

$$
\begin{aligned}
& T_{1}^{\mathrm{NL}, \mathrm{U}}=\int_{0}^{d_{1}} \exp \left(-\frac{\gamma P_{\mathrm{N}}^{\mathrm{U}}}{P^{\mathrm{D}} \zeta_{b_{o}}^{\mathrm{B} 2 \mathrm{U}}}\right) \mathscr{L}_{I_{\mathrm{agg}}^{\mathrm{U}, \mathrm{D}}}\left(\frac{\gamma}{P^{\mathrm{U}} \zeta_{b_{o}}^{\mathrm{B} 2 \mathrm{U}, \mathrm{NL}}}\right) \\
& \times \mathscr{L}_{I_{\mathrm{agg}}^{\mathrm{U}, \mathrm{U}}}\left(\frac{\gamma}{P^{\mathrm{U}} \zeta_{b_{o}}^{\mathrm{B} 2 \mathrm{U}, \mathrm{NL}}}\right) f_{R, 1}^{\mathrm{NL}}(r) d r,
\end{aligned}
$$

where the Laplace transform $\mathscr{L}_{I_{\mathrm{agg}}^{\mathrm{U}, \mathrm{D}}}(s)$ evaluated at $s=$ $\frac{\gamma}{P^{\mathrm{U}} \zeta_{b_{o}}^{\mathrm{B2U}, \mathrm{NL}}}$ can be derived as (55).

$T_{2}^{\mathrm{L}, \mathrm{U}}$ of $U L$

The result of $T_{2}^{\mathrm{L}, \mathrm{U}}$ is the UL coverage probability when the typical UE is associated with the typical BS with a LoS link of distance larger than $d_{1}$. The derivation of $T_{2}^{\mathrm{L}, \mathrm{U}}$ is very similar to $T_{2}^{\mathrm{L}, \mathrm{D}}$ and can be derived as

$$
T_{2}^{\mathrm{L}, \mathrm{U}}=0 \text {. }
$$

$T_{2}^{\mathrm{NL}, \mathrm{U}}$ of $U L$

Lemma 15. The UL coverage probability when $r>d_{1}$ and the signal is NLoS can be derived as

$$
\begin{aligned}
& T_{2}^{\mathrm{NL}, \mathrm{U}}=\int_{d_{1}}^{\infty} \exp \left(-\frac{\gamma P_{\mathrm{N}}^{\mathrm{D}}}{P^{\mathrm{D}} \zeta_{b_{o}}^{\mathrm{B} 2 \mathrm{U}}}\right) \mathscr{L}_{I_{\mathrm{agg}}^{\mathrm{U}, \mathrm{D}}}\left(\frac{\gamma}{P^{\mathrm{U}} \zeta_{b_{o}}^{\mathrm{B} 2 \mathrm{U}, \mathrm{NL}}}\right) \\
& \times \mathscr{L}_{I_{\mathrm{agg}}^{\mathrm{U}, \mathrm{U}}}\left(\frac{\gamma}{P^{\mathrm{U}} \zeta_{b_{o}}^{\mathrm{B} 2 \mathrm{U}, \mathrm{NL}}}\right) f_{R, 2}^{\mathrm{NL}}(r) d r,
\end{aligned}
$$

where the Laplace transform $\mathscr{L}_{I_{\mathrm{agg}}^{\mathrm{U}, \mathrm{D}}}(s)$ of $I_{\mathrm{agg}}^{\mathrm{U}, \mathrm{D}}$ evaluated at $s=\frac{\gamma}{P^{\mathrm{U}} \zeta_{b_{o}}^{\mathrm{B} 2 \mathrm{U}, \mathrm{NL}}}$ can be computed as

$$
\mathscr{L}_{I_{\mathrm{agg}}^{\mathrm{U}, \mathrm{D}}}(s)=\exp \left(-2 \pi p^{\mathrm{D}} \lambda \int_{r}^{\infty}\left[\frac{1}{1+\left(s P^{\mathrm{D}} \zeta^{B 2 B, N L}\right)^{-1}}\right] x d x\right) .
$$

\section{REFERENCES}

[1] D. López-Pérez, M. Ding, H. Claussen, and A. H. Jafari, "Towards $1 \mathrm{Gbps} / \mathrm{UE}$ in Cellular Systems: Understanding Ultra-Dense Small Cell Deployments," IEEE Communications Surveys Tutorials, vol. 17, pp. 2078-2101, Fourthquarter 2015.

[2] 3GPP, "TR 36.828 (V11.0.0): Further enhancements to LTE Time Division Duplex (TDD) for Downlink-Uplink (DL-UL) interference management and traffic adaptation," June 2012.

[3] M. Ding, D. López-Pérez, R. Xue, A. V. Vasilakos, and W. Chen, "On dynamic time-division-duplex transmissions for small-cell networks," IEEE Transactions on Vehicular Technology, vol. 65, pp. 8933-8951, Nov 2016.

[4] S. Goyal, C. Galiotto, N. Marchetti, and S. Panwar, "Throughput and coverage for a mixed full and half duplex small cell network," in 2016 IEEE International Conference on Communications (ICC), pp. 1-7, May 2016.
[5] M. Ding, D. López-Pérez, G. Mao, and Z. Lin, "What Is the True Value of Dynamic TDD: A MAC Layer Perspective," accepted to appear in IEEE Globecom 2017.

[6] B. Yu, L. Yang, H. Ishii, and S. Mukherjee, "Dynamic TDD support in macrocell-assisted small cell architecture," IEEE Journal on Selected Areas in Communications, vol. 33, pp. 1201-1213, jun 2015.

[7] A. K. Gupta, M. N. Kulkarni, E. Visotsky, F. W. Vook, A. Ghosh, J. G. Andrews, and R. W. Heath, "Rate analysis and feasibility of dynamic TDD in 5g cellular systems," in 2016 IEEE International Conference on Communications (ICC), Institute of Electrical and Electronics Engineers (IEEE), may 2016.

[8] H. Sun, M. Sheng, M. Wildemeersch, T. Q. S. Quek, and J. Li, "Traffic adaptation and energy efficiency for small cell networks with dynamic TDD," IEEE Journal on Selected Areas in Communications, vol. 34, pp. 3234-3251, dec 2016.

[9] Y. Zhong, P. Cheng, N. Wang, and W. Zhang, "Dynamic tdd enhancement through distributed interference coordination," in 2015 IEEE International Conference on Communications (ICC), pp. 3509-3515, June 2015.

[10] M. N. Kulkarni, J. G. Andrews, and A. Ghosh, "Performance of dynamic and static TDD in self-backhauled mmwave cellular networks," CoRR vol. abs/1701.07111, 2017.

[11] M. Ding, D. López-Pérez, A. V. Vasilakos, and W. Chen, "Analysis on the sinr performance of dynamic tdd in homogeneous small cell networks," in 2014 IEEE Global Communications Conference, pp. 15521558, Dec 2014.

[12] H. H. Yang, G. Geraci, Y. Zhong, and T. Q. S. Quek, "Packet throughput analysis of static and dynamic tdd in small cell networks," IEEE Wireless Communications Letters, vol. 6, pp. 742-745, Dec 2017.

[13] H. Sun, M. Wildemeersch, M. Sheng, and T. Q. S. Quek, "D2d enhanced heterogeneous cellular networks with dynamic tdd," IEEE Transactions on Wireless Communications, vol. 14, pp. 4204-4218, Aug 2015.

[14] M. Ding, D. López-Pérez, G. Mao, and Z. Lin, "Performance impact of idle mode capability on dense small cell networks," IEEE Transactions on Vehicular Technology, vol. 66, pp. 10446-10460, Nov 2017.

[15] S. Lee and K. Huang, "Coverage and economy of cellular networks with many base stations," IEEE Communications Letters, vol. 16, pp. 10381040, Jul. 2012

[16] M. Ding, D. López-Pérez, G. Mao, and Z. Lin, "Study on the idle mode capability with LoS and NLoS transmissions," in IEEE Globecom 2016, pp. 1-6, Dec. 2016.

[17] M. Ding, P. Wang, D. López-Pérez, G. Mao, and Z. Lin, "Performance Impact of LoS and NLoS Transmissions in Dense Cellular Networks," IEEE Transactions on Wireless Communications, vol. 15, pp. 23652380, March 2016.

[18] M. Ding and D. López-Pérez, "On the performance of practical ultradense networks: The major and minor factors," in 2017 15th International Symposium on Modeling and Optimization in Mobile, Ad Hoc, and Wireless Networks (WiOpt), pp. 1-8, May 2017.

[19] J.-S. Ferenc and Z. Néda, "On the size distribution of poisson voronoi cells," Physica A: Statistical Mechanics and its Applications, vol. 385, pp. 518-526, Nov 2007.

[20] S. Singh, H. S. Dhillon, and J. G. Andrews, "Offloading in heterogeneous networks: Modeling, analysis, and design insights," IEEE Transactions on Wireless Communications, vol. 12, pp. 2484-2497, May 2013.

[21] I. Gradshteyn and I. Ryzhik, Table of Integrals, Series, and Products (7th Ed.). Academic Press, 2007.

[22] M. Ding, D. López-Pérez, R. Xue, A. Vasilakos, and W. Chen, "On dynamic Time-Division-Duplex transmissions for small-cell networks," IEEE Transactions on Vehicular Technology, vol. 65, pp. 8933-8951, Nov. 2016.

[23] T. Ding, M. Ding, G. Mao, Z. Lin, D. López-Pérez, and A. Zomaya, "Uplink performance analysis of dense cellular networks with los and nlos transmissions," IEEE Transactions on Wireless Communications, vol. PP, no. 99, pp. 1-1, 2017. 